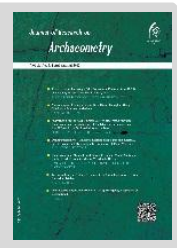

Original Paper

\title{
Identify Hidden Designs in Antique Paintings Using Industrial Radiography
}

\author{
Mahdi Mirzapour ${ }^{1 \star}$, Effat Yahaghi $^{2}$, Maryam Ghiasi Limenjubi ${ }^{3}$, Jose Antoni Madrid \\ Garcia $^{4}$, Amir Movafeghi ${ }^{5}$
}

\author{
${ }^{1}$ Assistant Professor, Department of Mathematics, Bu-Ali Sina University, Hamedan, IRAN \\ ${ }^{2}$ Associate Professor, Department of Physics, Imam Khomeini International University, Qazvin, IRAN \\ ${ }^{3}$ Student, Department of Physics, Imam Khomeini International University, Qazvin, IRAN \\ ${ }^{4}$ Professor, University Institute of Repair, Valencia Polytechnic University, Valencia, SPAIN \\ ${ }^{5}$ Associate Professor, Reactor and Nuclear Safety Research School, Nuclear Science and Technology Research \\ Institute, Tehran, IRAN
}

\begin{abstract}
In past centuries, to prevent exquisite paintings, new works of art were painted on them. To identify the hidden paintings on the board, using digital radiography as a non-destructive testing method is recommended. Some phenomenon such as photon scattering, different types of noises, etc. causes on the quality of output radiographs. In this paper, we use two pyramid-based techniques, i.e., the Gaussian pyramid method and the Laplacian pyramid method, to improve the quality of radiographs. The experimental results show the effectiveness of applied techniques for identifying the hidden paintings.

Keywords: Hidden designs, Antique paintings, Industrial radiography, Image processing, Gaussian Pyramid Method, Laplacian Pyramid Method.
\end{abstract}

\section{Introduction}

Artwork in museums is a major source of scientific, historical, and other research related to archeology. Different risks such as rupture, discoloration, and biodegradation threaten the paintings. Insect's attack, natural disasters, and temperature changes are some other risk factors [13].

Preservation of art pieces is an essential and challenging task during wars and different social events. In the past, new works of art were painted on existing paintings to prevent the burning of exquisite ones that were not compatible with the post-war social conditions [4-7]. Examples are including portraits of celebrities and leaders. This could also be done due to a lack of raw materials and the obsolescence of many paintings. In some cases, these works were painted by various artists, and there is no information about the process of their changes. There are various methods for detecting defects and hidden designs of boards [6-10]. Regarding the materials used in different layers that are different in each historical period, useful information can be obtained about the designs and other artwork properties. An important issue in art paintings is their preservation and identification of defects without damaging the painting. Various non-destructive techniques are used for this purpose [11-15]. Radiographic examination is an important part of non-destructive testing [16-19]. In radiography, latent patterns and serious damage are revealed due to the

\footnotetext{
* Corresponding author: m.mirzapour@basu.ac.ir

Copyright@ 2020, the Authors / This open-access article is published under the terms of the Creative Commons Attribution-NonCommercial 4.0 International License which permits Share (copy and redistribute the material in any medium or format) and Adapt (remix, transform, and build upon the material) under the Attribution NonCommercial .terms
} 
penetration of X-rays into the underlying layers. X-rays can reveal the hidden image of a painting on different layers [20-22]. Before using the radiographic test, the thickness of the painting layers leads the experts to believe that the painting is not the original and another design has been drawn on it. Radiographic images may suffer from the photons scattering, different noises, geometrical unsharpness, and blurring effects. The quality of the radiographic images can be reduced by those phenomena. Image processing methods can be used to increase the quality of radiographs [23-27].

\section{Methods}

Gaussian and Laplacian Pyramids methods

In this research, by using the sub-sampling approach, we constructed an image pyramid, e.g., Fig. 1A, which leads to two pyramid-based methods, namely the Gaussian pyramid and the Laplacian pyramid [28,29]. The Laplacian pyramid will be construct based on the Gaussian pyramid, i.e., there is a Relationship between Gaussian and Laplacian Pyramids, see Fig. 1-B for more details. The mentioned methods are user-friendly (due to the small number of input parameters), easy to implement and also have low computational cost [28]. Therefore, these methods can be used to improve the quality and reduce the opacity of radiographs. In the following, we briefly describe these methods. We begin with the Gaussian pyramid. Let $I=g_{0}$ be the input noisy image, i.e., the base of the pyramid. Each layer in the pyramid will be constructed by a reduction operator in the following form

$$
\begin{gathered}
\boldsymbol{g}_{\boldsymbol{\ell}}(\boldsymbol{i}, \boldsymbol{j})=\sum_{\boldsymbol{m}=-\mathbf{2}}^{\mathbf{2}} \sum_{\boldsymbol{n}=-\mathbf{2}}^{\mathbf{2}} \boldsymbol{w}(\boldsymbol{m}, \boldsymbol{n}) \boldsymbol{g}_{\boldsymbol{\ell}-\mathbf{1}}(\mathbf{2 i}+\boldsymbol{m}, \mathbf{2} \boldsymbol{j}-\boldsymbol{n}), \\
\text { where } \boldsymbol{w}=\widehat{\boldsymbol{w}} \times \widehat{\boldsymbol{w}}^{\boldsymbol{T}} \text { is a Gaussian window (filter). In this research, we set } \\
\widehat{\boldsymbol{w}}^{\boldsymbol{T}}=\left[\begin{array}{ll}
\mathbf{0} .0625 & 0.250 .3750 .250 .0625
\end{array}\right]
\end{gathered}
$$

It is known that the Laplacian pyramid provides an extra level of analysis as compared to the Gaussian pyramid by breaking the image into different isotropic spatial frequency bands [28]. Assume that $\boldsymbol{g}_{\boldsymbol{\ell}}$ and $\boldsymbol{g}_{\boldsymbol{\ell}+\mathbf{1}}$ represent two successive layers (scales) of the Gaussian pyramid. The $\ell^{\text {th }}$ layer of the Laplacian pyramid is defined in the following form

$$
L_{\ell}=g_{\ell}-\operatorname{expand}\left(g_{\ell+1}\right)
$$

where the operator $\boldsymbol{e x p a n d}\left(\boldsymbol{g}_{\boldsymbol{\ell}+\mathbf{1}}\right)$ up-samples layer $\boldsymbol{g}_{\boldsymbol{\ell}+\mathbf{1}}$ by doubling its size and defined as follows

$$
\operatorname{expand}\left(g_{\ell+1}(i, j)\right)=4 \sum_{m=-2}^{2} \sum_{n=-2}^{2} w(m, n) g_{\ell+1}\left(\frac{i-m}{2}, \frac{j-n}{2}\right)
$$

Therefore, the denoised image can be obtained in the following form

$$
\widehat{g}_{0}=L_{0}+\operatorname{expand}\left(g_{1}\right) \text {. }
$$

In this paper, to improve the contrast of radiographs and enhance their qualities, we use the background removal technique. In this technique, we consider the $\ell^{t h}$ layer of an arbitrary pyramid, and using (3) we change its size to the size of original input image $I$, then by subtracting it from the original image, we obtain the denoised image $\boldsymbol{I}_{\text {denoised }}$.

The Gaussian pyramid method and the Laplacian pyramid method are summarized in Algorithm 1 and 2, respectively.

Algorithm 1: The background removal technique using the Gaussian pyramid method

1. Inputs:

- $\quad$ Noisy image $\boldsymbol{I}=\boldsymbol{g}_{\mathbf{0}}$

- Number of layers $\boldsymbol{S}$

- Filter $\boldsymbol{w}$

2. For $\boldsymbol{\ell}=\mathbf{0}, \mathbf{1}, \ldots, \boldsymbol{S}$ do

- Compute the $\boldsymbol{\ell}^{\text {th }}$ layer of the Gaussian pyramid using (1)

3. Using expand operator (3) set

4. Set

$$
\widehat{g}_{0}=\operatorname{expand}\left(g_{S}\right)
$$

$I_{\text {denoised }}=I-\widehat{g}_{0}$. 
Algorithm 2: The background removal technique using the Laplacian pyramid method

1. Inputs:

- $\quad$ Noisy image $\boldsymbol{I}=\boldsymbol{g}_{\mathbf{0}}$

- Number of layers $\boldsymbol{S}$

- Filter $\boldsymbol{w}$

2. For $\boldsymbol{\ell}=\mathbf{0}, \mathbf{1}, \ldots, \boldsymbol{S}$ do

- Compute the $\ell^{\text {th }}$ layer of the Gaussian pyramid using (1).

- Compute the $\ell^{t h}$ layer of the Laplacian pyramid using (2) and (3).

3. Using expand operator (3) set

$$
\widehat{L}_{0}=\operatorname{expand}\left(L_{S}\right)
$$

4. Set

$$
I_{\text {denoised }}=I-\widehat{L}_{0} .
$$

For evaluating the reconstructed images and their similarity, the peak signal-to-noise ratios (PSNR) and mean squared error (MSE) of images are calculated. Given an original image $I \in$ $\mathbb{R}^{M \times N}$ and its reconstructed image $\mathrm{I}_{\text {denoised }} \in \mathbb{R}^{M \times N}$, PSNR and MSE are defined as [29]:

and

$$
\operatorname{PSNR}=\frac{1}{M N} \log \left(\frac{M N}{\sum_{i=1}^{M} \Sigma_{j=1}^{N}\left(I(i, j)-I_{\text {denoised }}(i, j)\right.}\right),
$$

$$
\operatorname{MSE}=\sqrt{\frac{1}{M N} \sum_{\mathbf{i}=1}^{M} \sum_{j 1}^{N}\left(I(i, j)-I_{\text {denoised }}(\mathbf{i}, \mathbf{j})\right)^{2}}
$$

\section{The radiography procedure}

The employed X-ray unit is the TRANXPORTIX 50 X-ray machine made by General Electric ${ }^{\circledR}$. It has $3 \mathrm{~kW}$ output power, a focal spot size of $2.3 \mathrm{~mm}$, and a total filtration of $2 \mathrm{~mm}$ of aluminum. The range of high voltage variation is between $20 \mathrm{kVolt}$ to $110 \mathrm{kVolt}$. The phosphor imaging plates (computed radiography, CR) and conventional industrial radiography films (AGFA® films) are used for X-ray detection and image generation.

\section{Results}

The X-ray radiography technique was used to examine hidden designs in valuable paintings. In Fig. 2 , the radiographs of paintings of Fig. 1 are shown. It is noticeable that the designs of radiographs are completely different from the design on the canvas or the woods. Besides, the hidden construction detail, defects and damage of painting can also be recognized in radiography of paintings. Therefore, the radiography technique can reveal the hidden designs and help the restorer for better decision. The quality obtained hidden radiographs have been suffering from some blurriness. Digital image processing techniques has been implemented to enhance the quality of the images. For this purpose, the Gaussian and Laplacian Pyramids methods were applied to the images to improve the quality of assessment of hidden paintings. Fig. 4 shows the output of the Gaussian and Laplacian Pyramids methods for Fig. 2-A. It is difficult to find the best threshold level for an unknown dynamic noise and to remove the foggy component of the images. Therefore, a method of the background removal has been utilized. According to Figs. 4 and 5, the high levels of decomposition in the sub-sampling approach are caused a very foggy output images. We subtracted the original radiograph (Fig. 2-A) to the foggy component (Fig.4). The reconstructed images have higher contrast and sharper edges. The reconstructed images in this way are shown in Figs. 6-8 for the burial of the sardine, the scenery and two ladies paintings. It can be seen that the reconstructed images are clearer and have sharper edges in the content of the paintings. Two radiography experts and two restoration specialists evaluated the results. Table 2 shows experts' scores for original and reconstructed radiographs by Gaussian pyramid and Laplace pyramid methods. The professionals' opinions confirm that the reconstructed images are clear and have visualized better than the original ones. 


\section{Conclusion}

The results show that radiography is an effective method in identifying hidden paintings. Digital processing of radiographic images can also help for better identification of hidden designs. The effectiveness of the Gaussian Pyramid is to decompose the original radiograph into smoother and to extract the feature or structure of interest, and it can also attenuate the image noise. This goal can be achieved by two simple operations, i.e., smoothing and down-sampling which the former process can smooth the images with a sequence of smoothing filters and the latter operation reduces image size by half after each smoothing. Also, levels of the Laplacian pyramid will be constructed to evaluate the difference between two successive levels of the Gaussian pyramid. When the level of the Gaussian/Laplacian pyramid is obtained, the improved radiograph will be constructed by subtracting its values from the original radiographs. The mentioned methods are implemented on the several painting of the Valencia Museum. The results show the effectiveness of the radiography along with digital image processing techniques for image enhancement of the paintings specimens. 


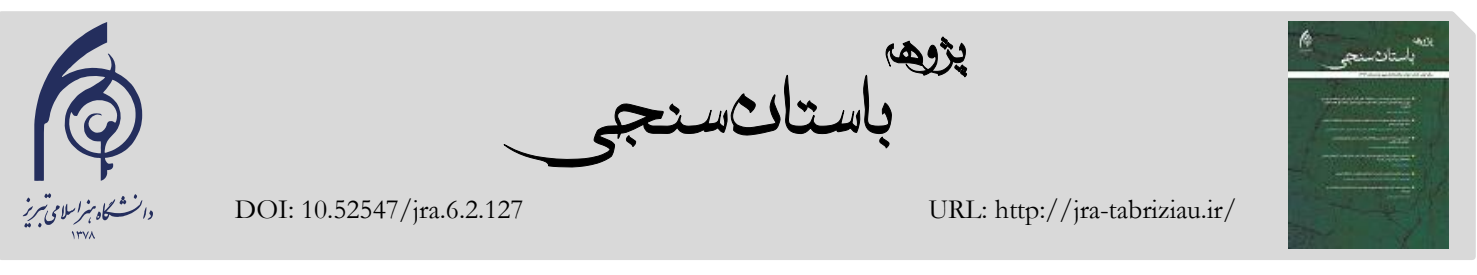

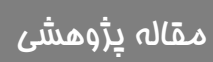

\section{Cب0ssnark}

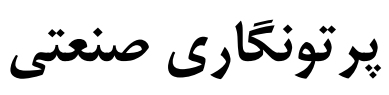

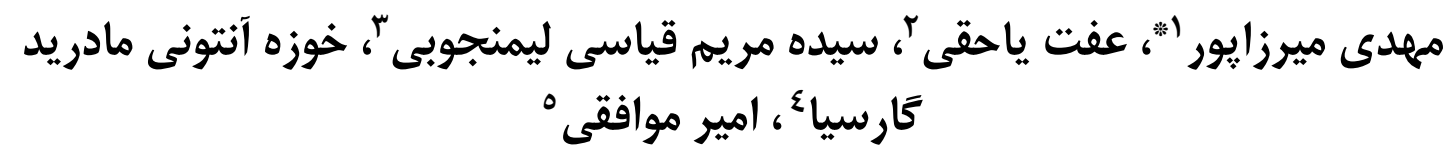

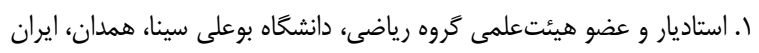

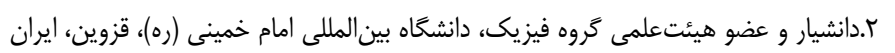

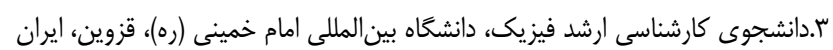

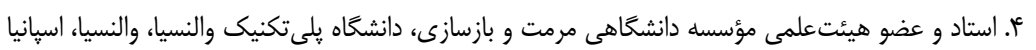

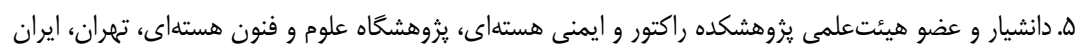

\section{جكيده}

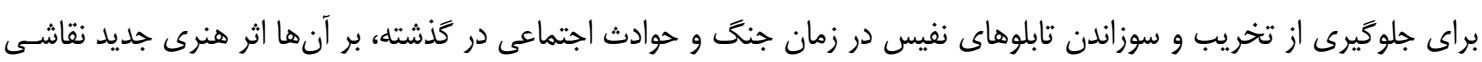

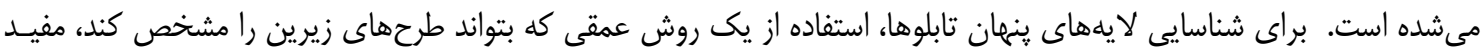

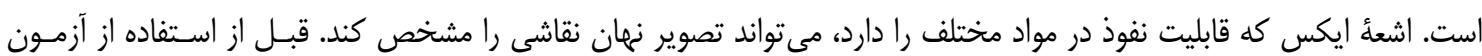

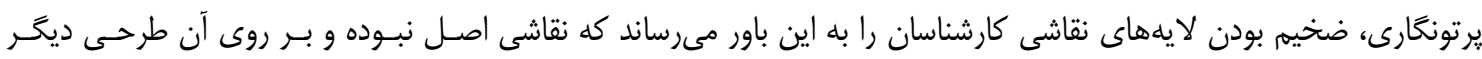

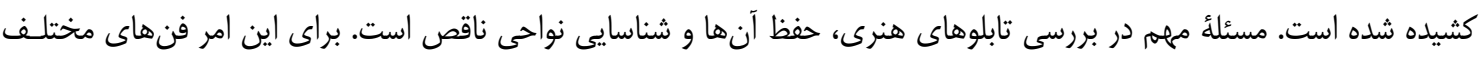

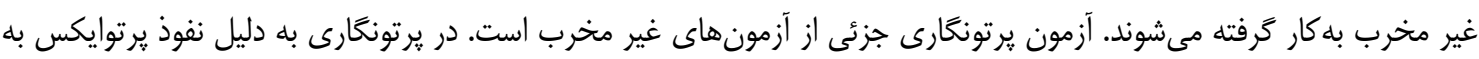

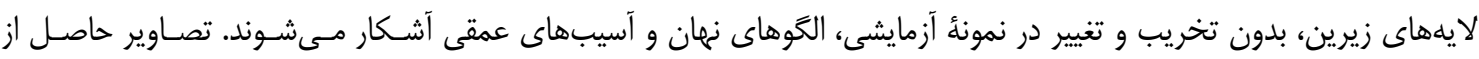

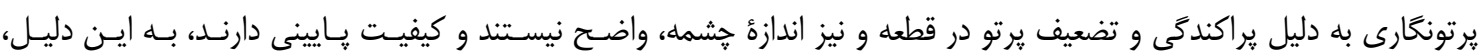

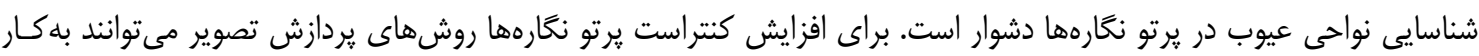

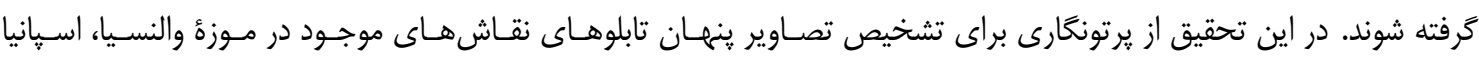

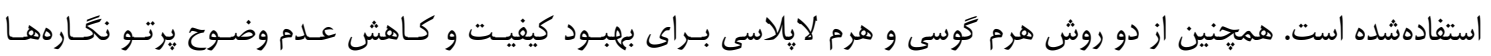

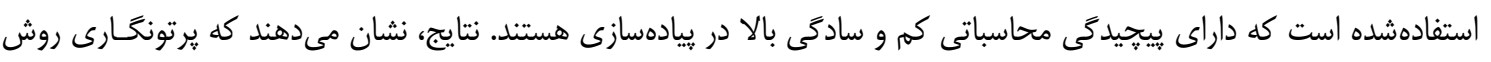

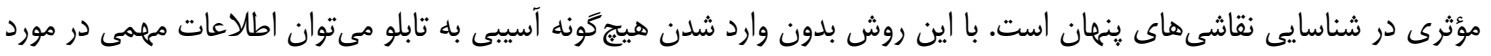

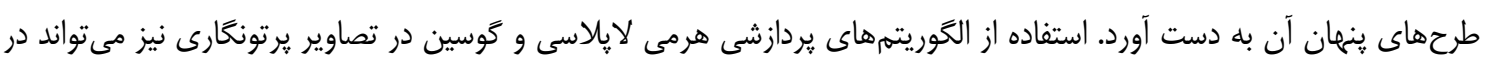

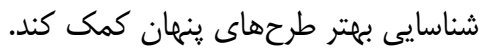

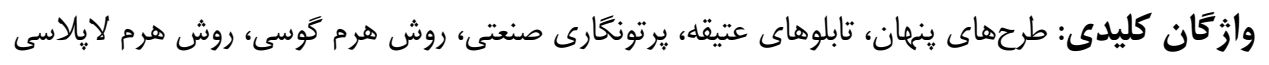

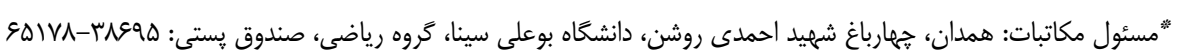
m.mirzapour@basu.ac.ir : 


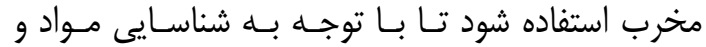

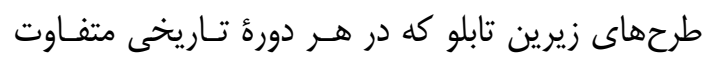

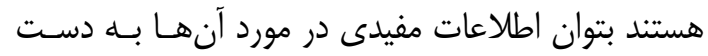
آورد [1,3,5].

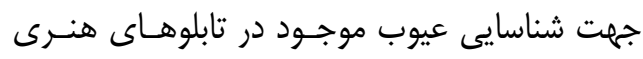

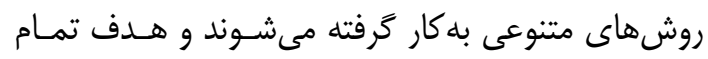

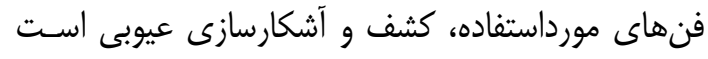

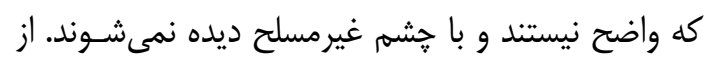

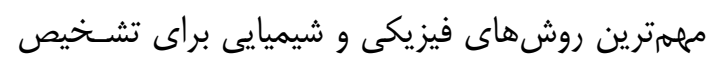

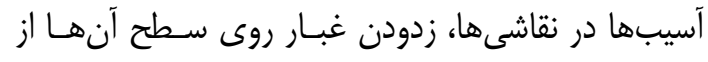
طريق مكش، ضدعفونى كردن، استفاده از آباكسيثنه و و

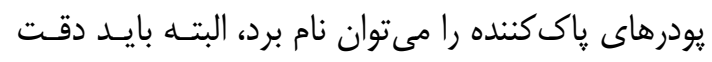
شود كه مواد شيميايى بلهمروززمان موجـب نـابودى بـ آثار

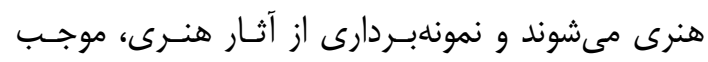
تخريب و از بين رفتن آنها مىشودي روشهاى ديكرى نيز براى شناسايى تخريب تابلوها

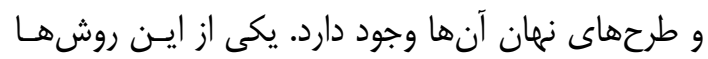

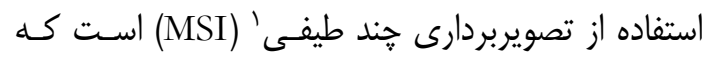

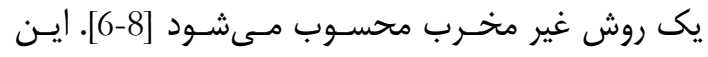

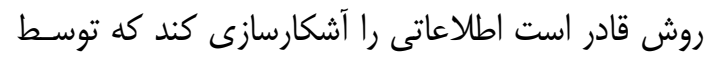

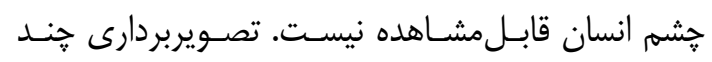

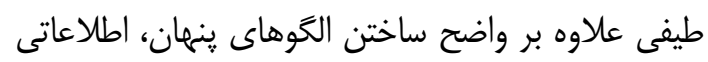

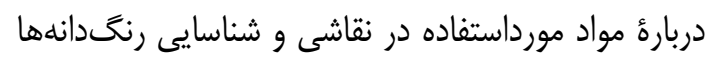

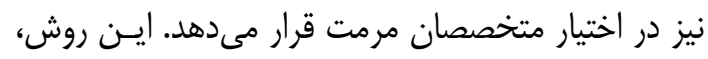

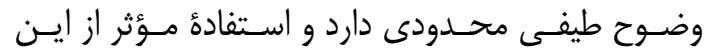

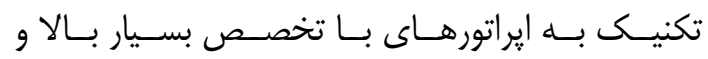
دستخاههاى مكانيكى ويزماى نياز دارد [12-9]. همجنينين

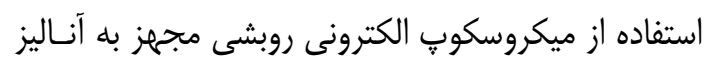
اسيكتروسكويى يراش انرزى يرتوايكس ' (SEM-EDS)

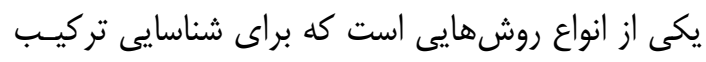

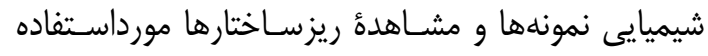
قرار مى يَيرد [13]. عالاوه بر روشهاى بالا، آزمايشهايى وجـود دارنس

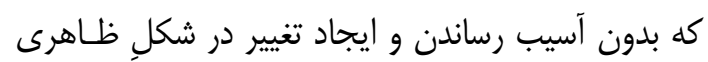

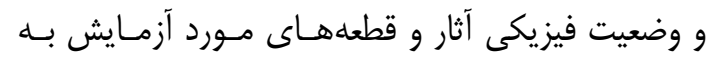

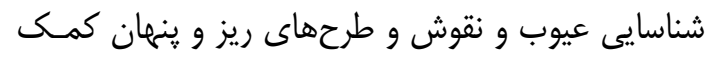

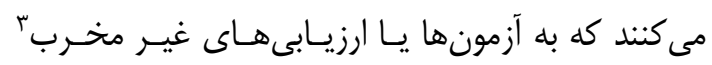

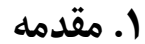

آثار هنرى و باستانى ازنظر مـادى، فرهنخـى و تـاريخى

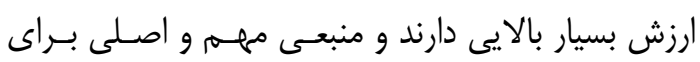

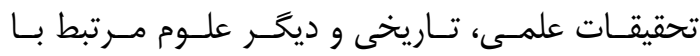

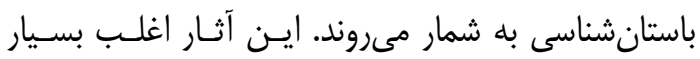

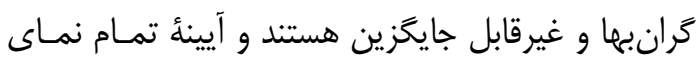

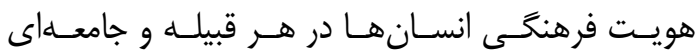

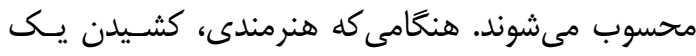

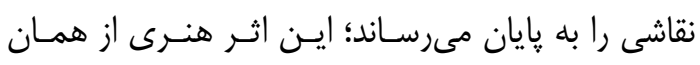
لحظه در معرض تغيير و زوال قـرار مسى يـيــد. تغييـرات

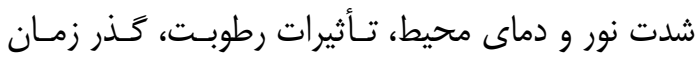
(ييرى طبيعى)، آسيبهاى تصادفى، آلـودگى باكتريـايى،

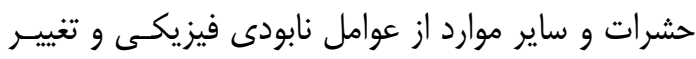

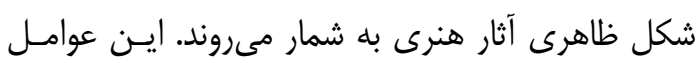
موجب ايجاد شورهزدمى در سطح نقاشى، گسترش ش و يـا

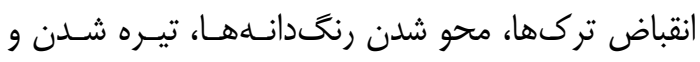

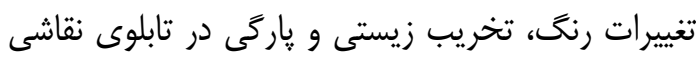

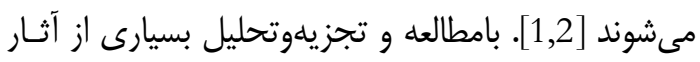

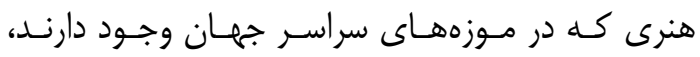

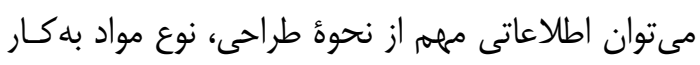

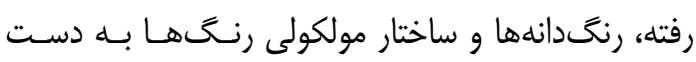

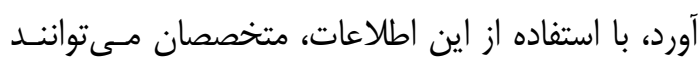
روشى خاص براى حفاظت و مرمت آثار ييشـنهاد كنتــد.

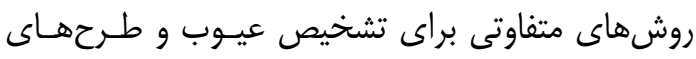

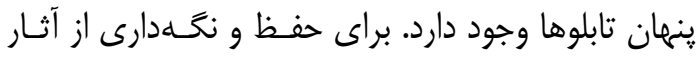

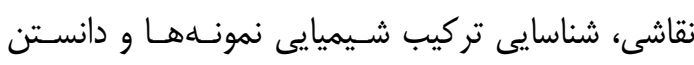

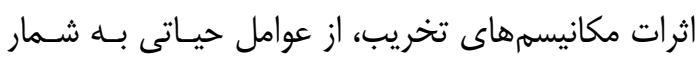

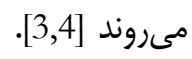
اگر آسيبهاى زيرسطحى و نقشهاى نهان موجود در تابلوى هنرى شناسايى نشوند و جلوى تخريب آنهـا

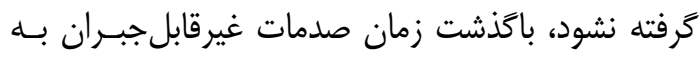

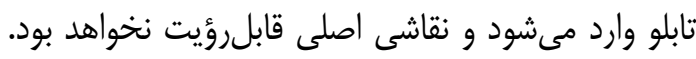

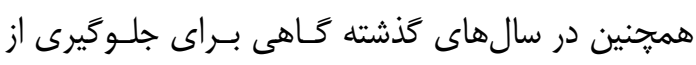

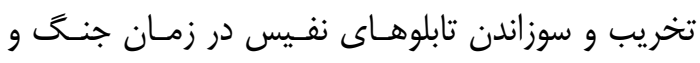

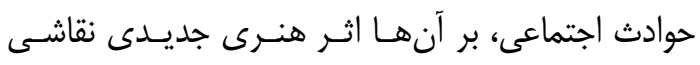

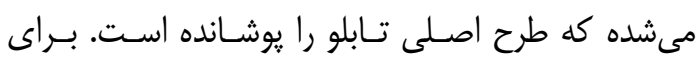

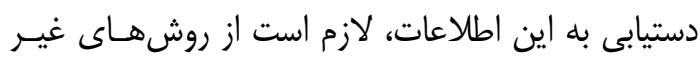


تفسير صحيح و مناسب يرتو نظارهها براى ارزيـابى و به دست آوردن اطلاعات بيشتر و دقيقتر جهت شناسـايى نئي

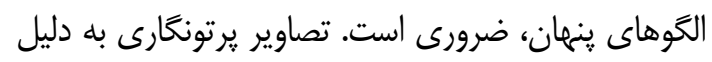

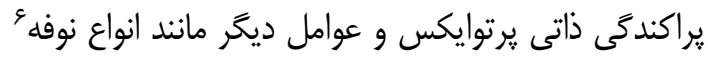

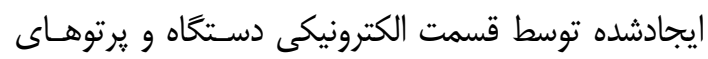

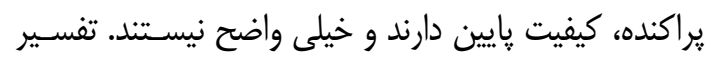

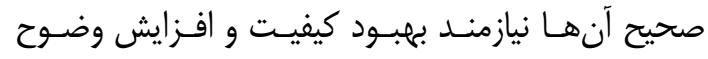

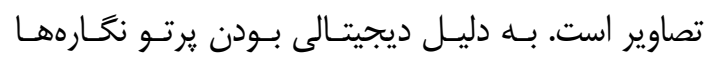

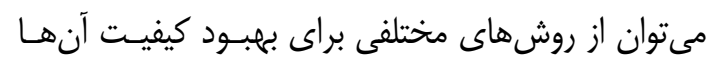

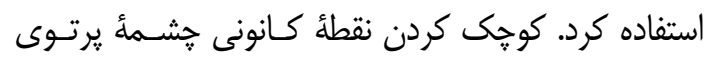

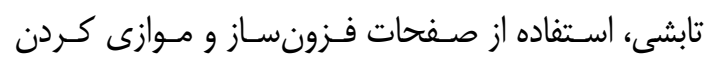

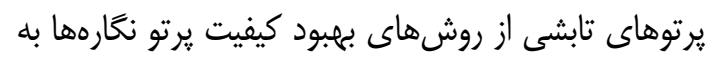

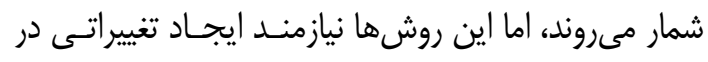

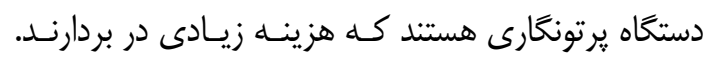

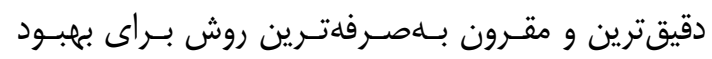

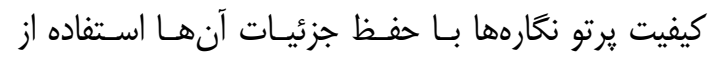

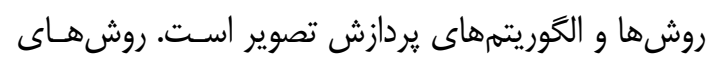

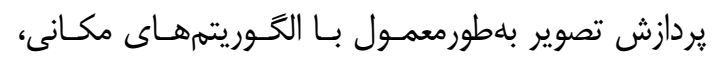

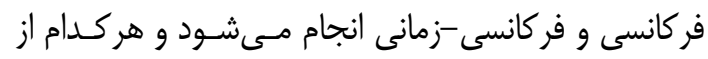

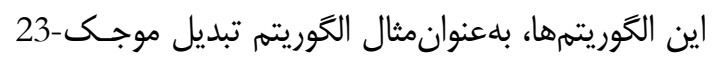

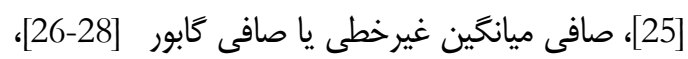

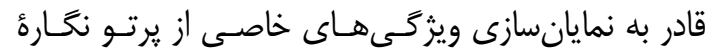
تابلوهاى نقاشى هستند.

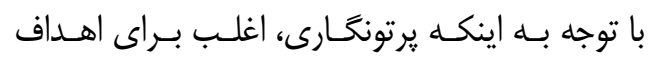

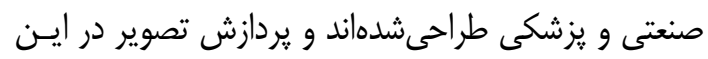

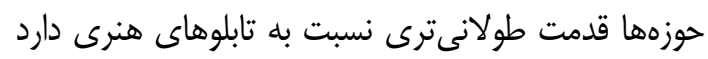

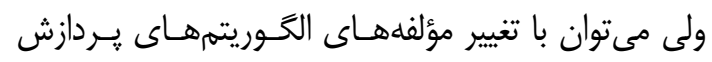
تصوير، كارايى آنها را براى تابلوهاى هنرى افزيايش دائ داد و

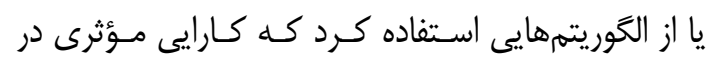

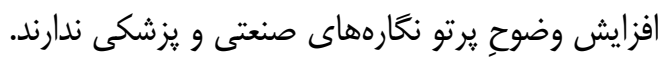
در اين تحقيق، براى بهبود كيفيت تصاوير برتونغارى

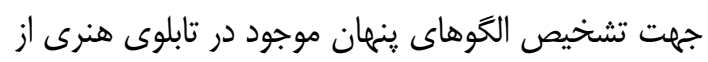

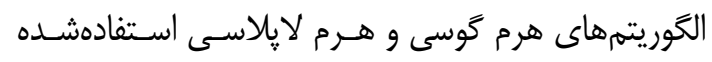
است. هدف از ساخت هر مهاى تصوير، نمـايش اسـتخراج

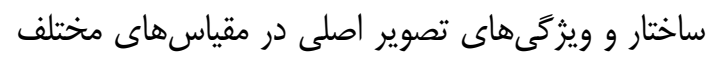

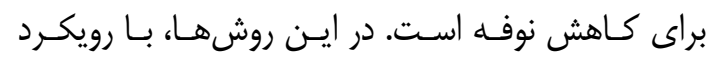

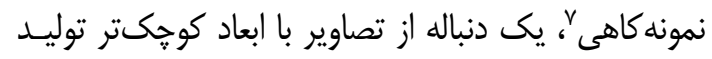

معـروف هسـتند. انــواع مختلــف (NDT or NDE)

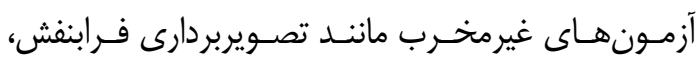

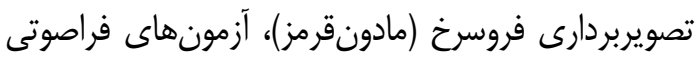

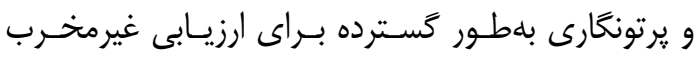
تابلوهاى هنرى شناختهشدهاند [11-8] و واستفاده مى شيوند.

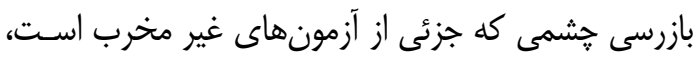

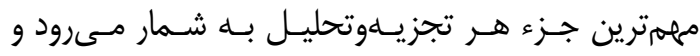

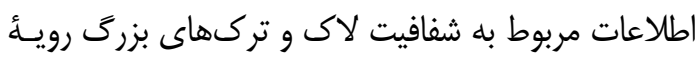

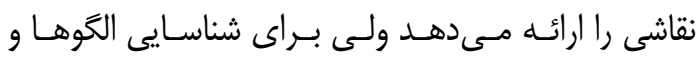

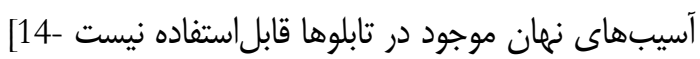

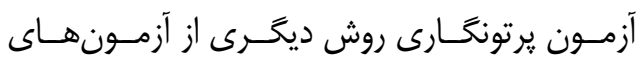

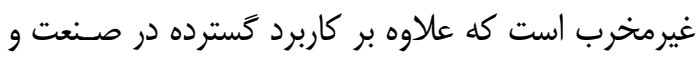
يزشكى، در علوم و مطالعات باستانشناسى براى شناسايى كاريى

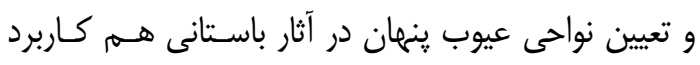

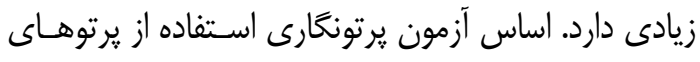

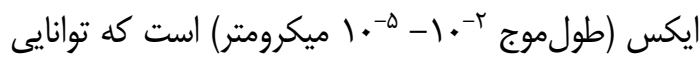

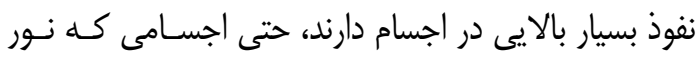

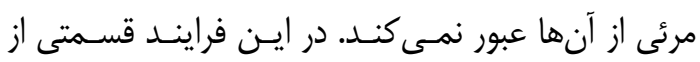

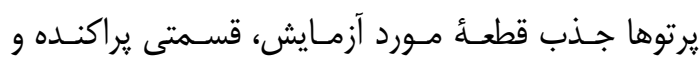

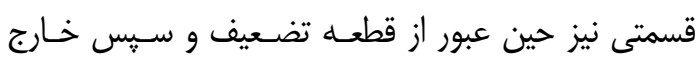

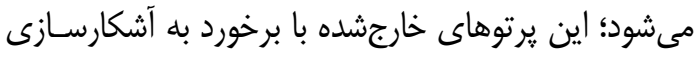

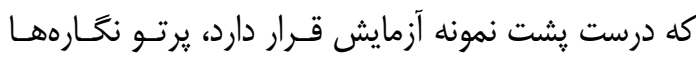

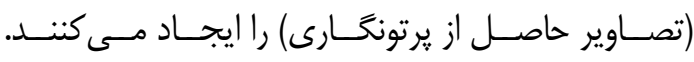

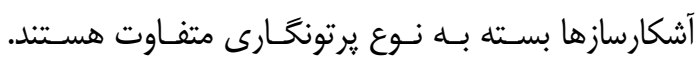

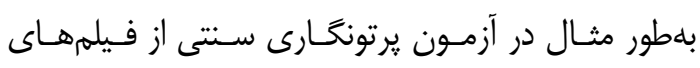

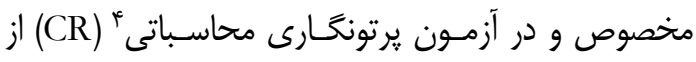
صفحات فسفرىه تصويرساز بلهنوان آشكارسـاز اسـتفاده مىشود [22-19]. با يرتونگارى تابلوهاى هنرى مى 19توان اطلاعاتى را در

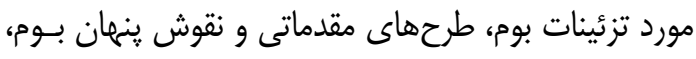

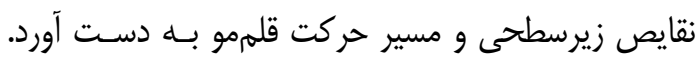

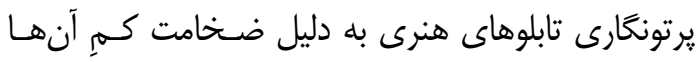

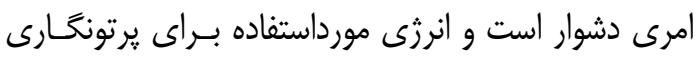

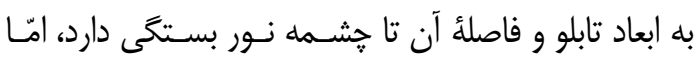

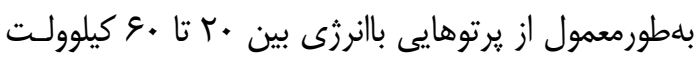
براى يرتونغارى استفاده مىشود. 
بيشترى دارد. پٍ از ساخت هرم، براى بازسـازى تصـوير

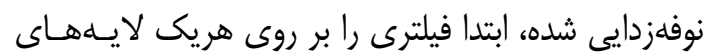

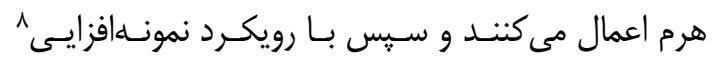

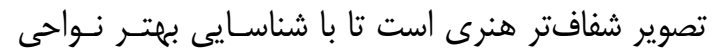

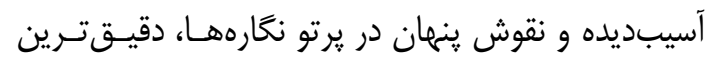

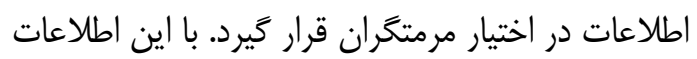

مىشوند كه به توليد هرم تصوير منجر مى شوند. در روش

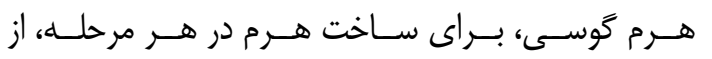

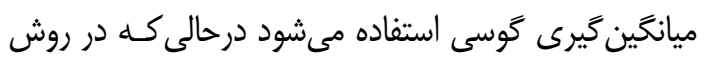

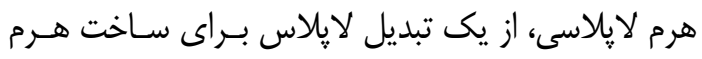

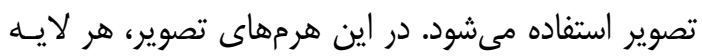

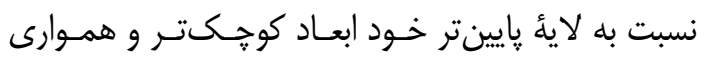

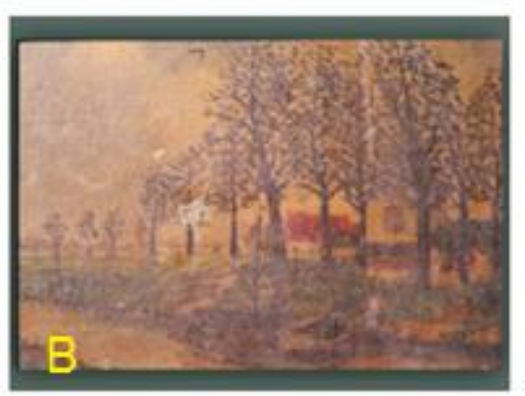

$\varphi$

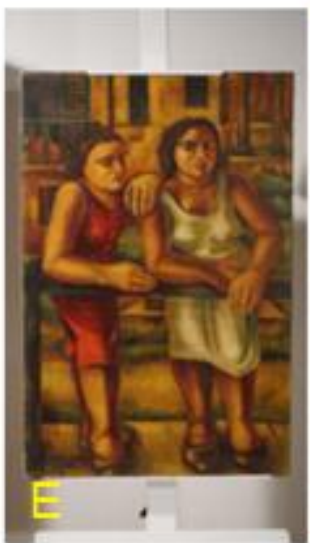

$\dot{\sim}$

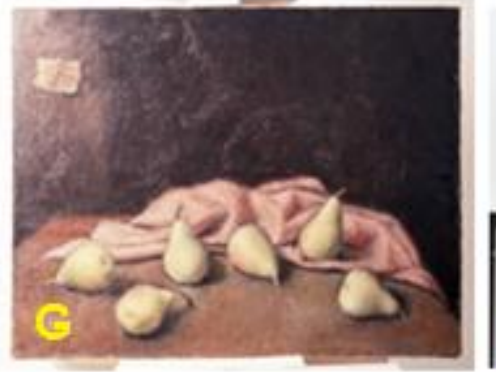

飞

$ت$

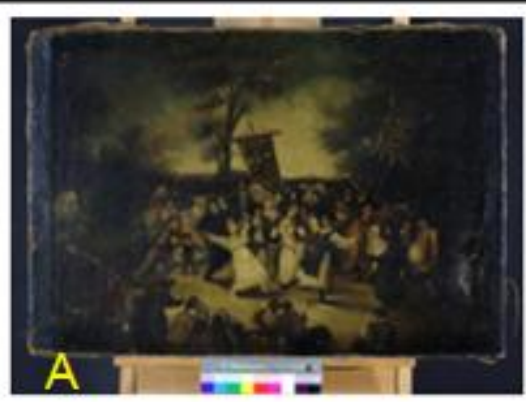

الف
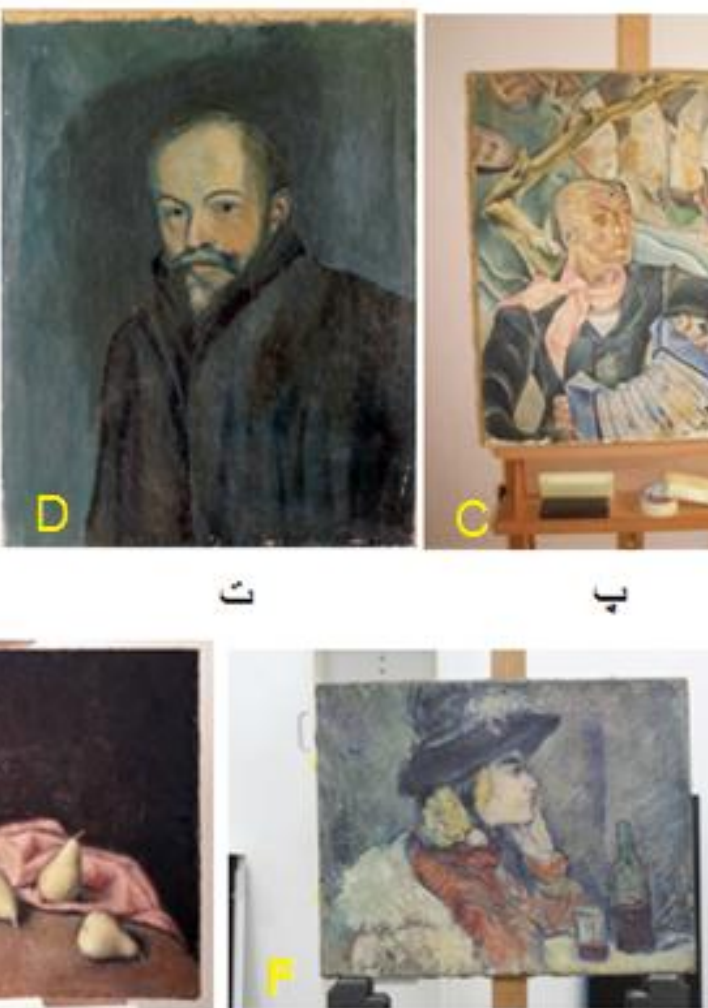

ج

شكل ا: تصاوير تابلوها الف- دفن ساردين، ب - جشه|نداز، پֶ- مردى با آكاردئون، ت- يرتره انسان، ث- دو زن ج- بانو، د- هنوز زندگى با كابى

Fig. 1: Painting: A(The burial of the sardine, B) Scenery, C) Man with Accordion, by Genaro Lahuerta, D(Portrait of man, E) Two ladies, F(Lady G) Still Life with Pears by Valentine Durban 
كه بعد از يرتو كيرى با اسكنر ليـزرى مخصـوص -CR 30

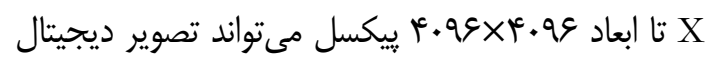

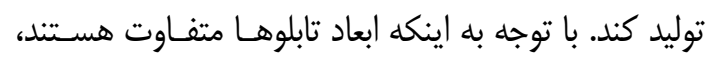

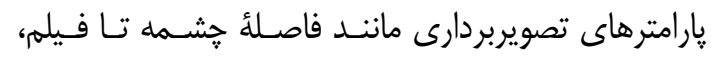

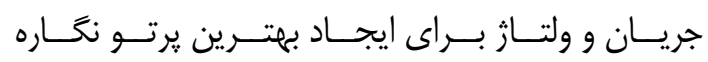

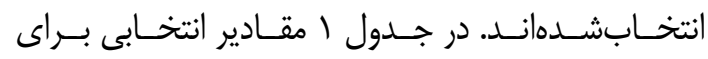
يرتونگارى هر تابلو نوشتهشده است.

\section{ץ-ץ. الخَوريتمهاى هرم تَوسى و هرم لايلاسى}

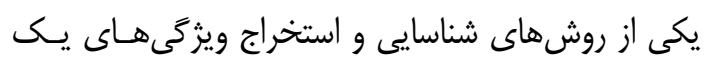

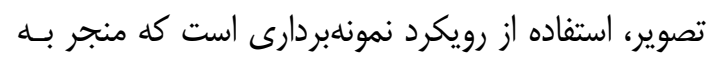

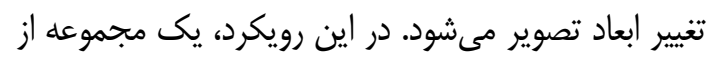

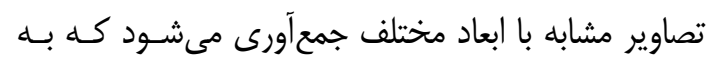

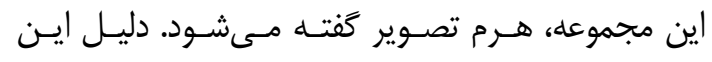

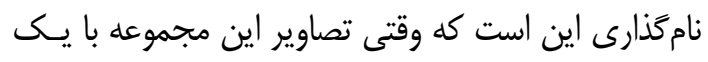

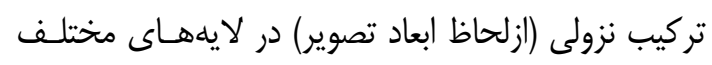

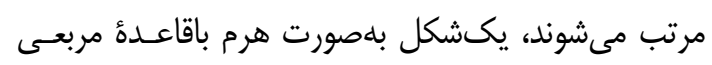
شكل ظاهر مىشود. شكل 1-الف بيانكر يك هرم تصوير

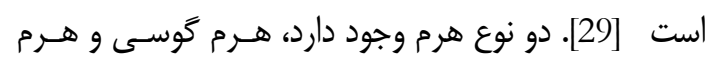

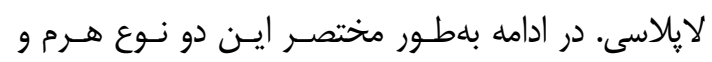
ارتباط بين آنها شرح داده مىشود. فرض كنيم تصوير نوفه

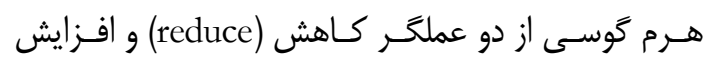

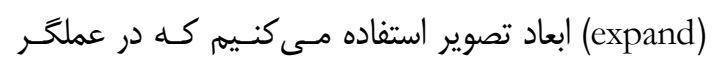

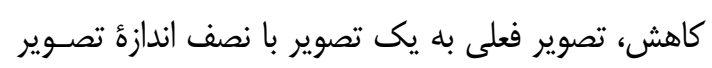

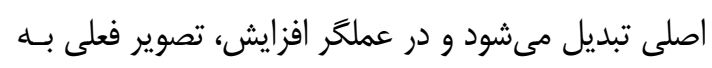

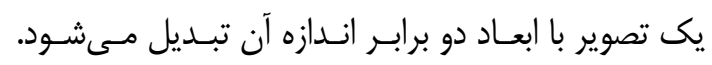
بنـابراين ســطح

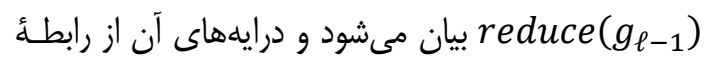
زير محاسبه مىشوند: $g_{\ell}(i, j)=\sum_{m=-2}^{2} \sum_{n=-2}^{2} w(m, n) g_{\ell-1}(2 i+$ $m, 2 j-n)$

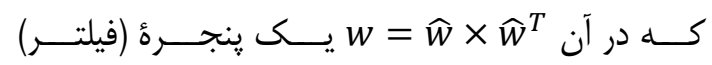
كوسى 5 × 5 است. ما در نتايج تجربى اين تحقيق قرار مى دهيث:

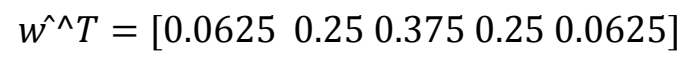
همانطور كه ملاحظه مى شود در هرم گوسى ابتدا
مرمتكران مىتوانند مناسبترين روش را براى تـرميم و

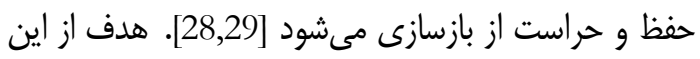

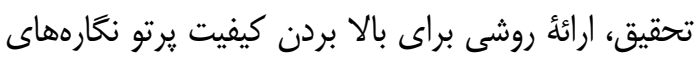

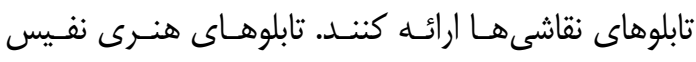
استفادهشه در اين تحقيق متعلق به موزه هنـــى والنسـيا

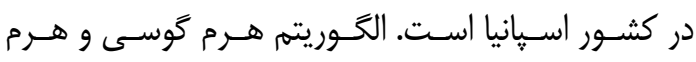

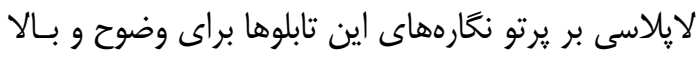

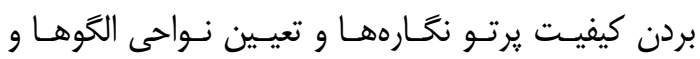
نقاشىهاى نهان اعمالشهد است.

\section{r. r- إ. معرفى تابلوها} هفت تابلو از آثار هنرى و تاريخى موزهُ والنسـيا در اســانيا

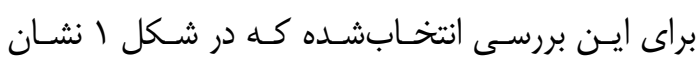

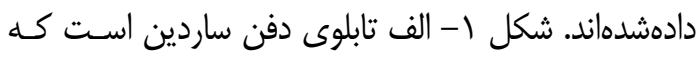

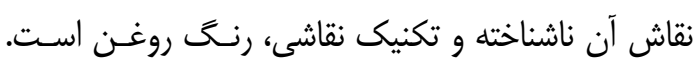

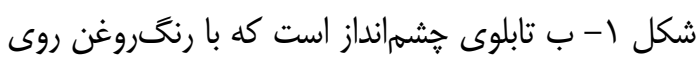

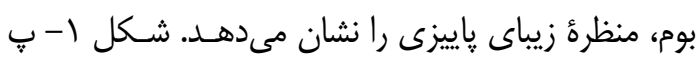

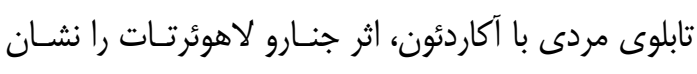

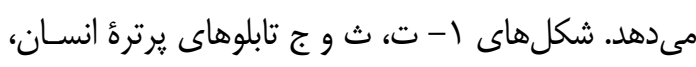

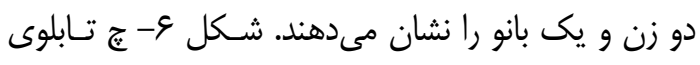

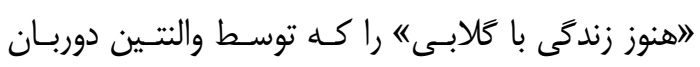
كشيده شده است، نشان مىدهد.

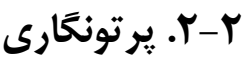
يرتونغارى با يكى دستخاه تـرانس يـورتيكس

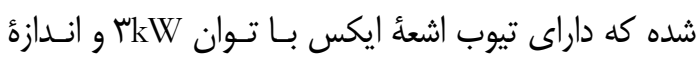

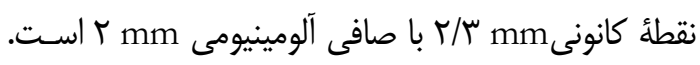

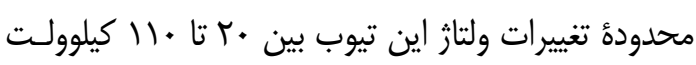
است. اين دستخاه مولد يرتوايكس، يك سيستم تابش نـور

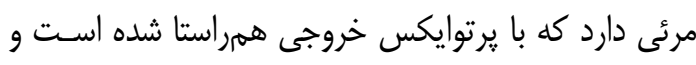

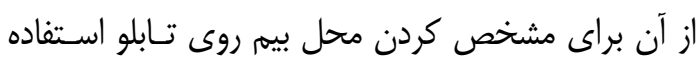

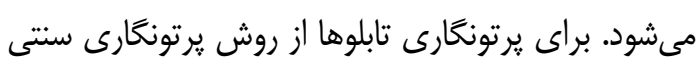

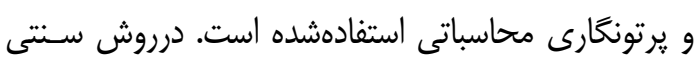

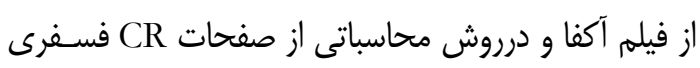

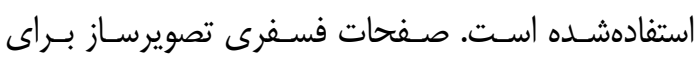
آشكارسازى اشعهُ ايكس عبورى از جسم استفادهشده است 

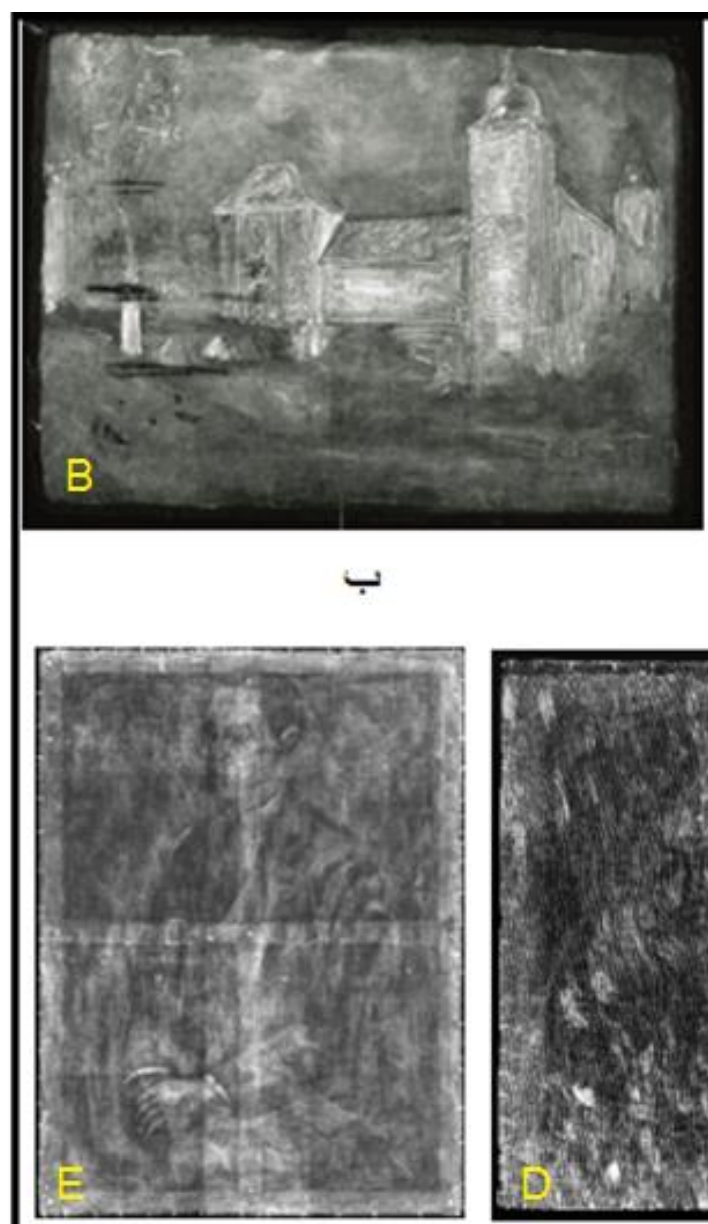

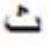

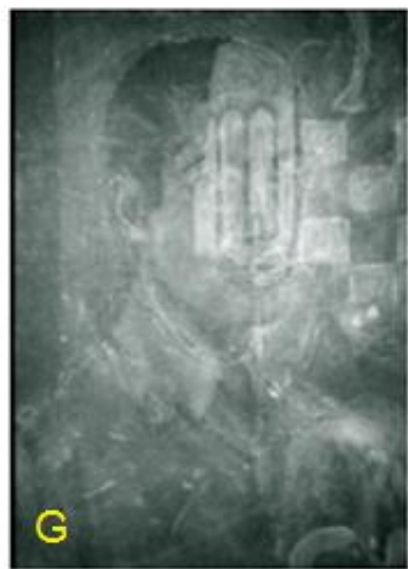

飞

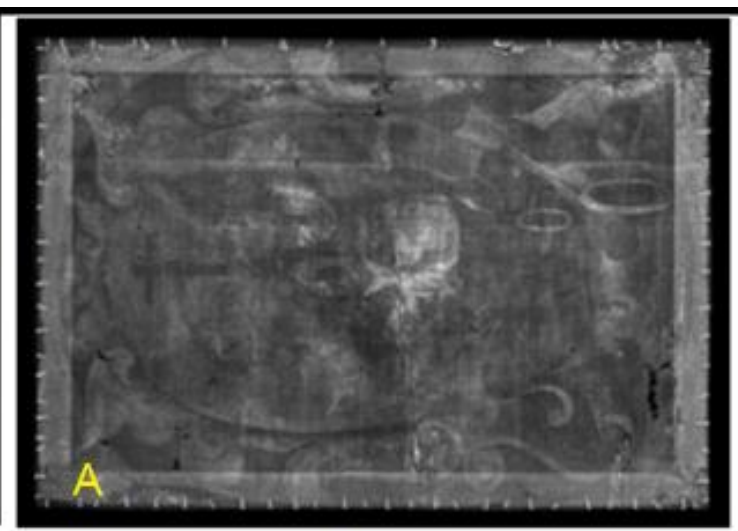

الف

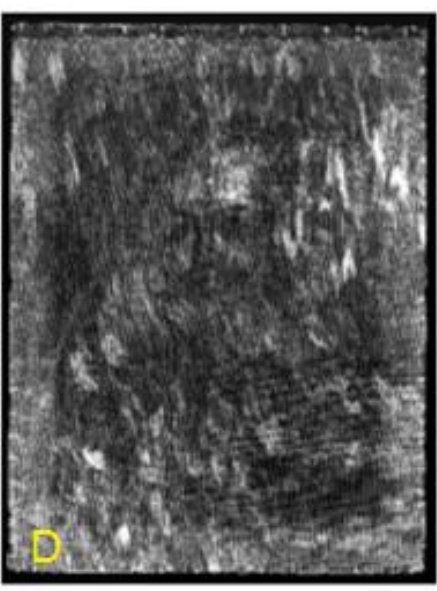

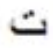

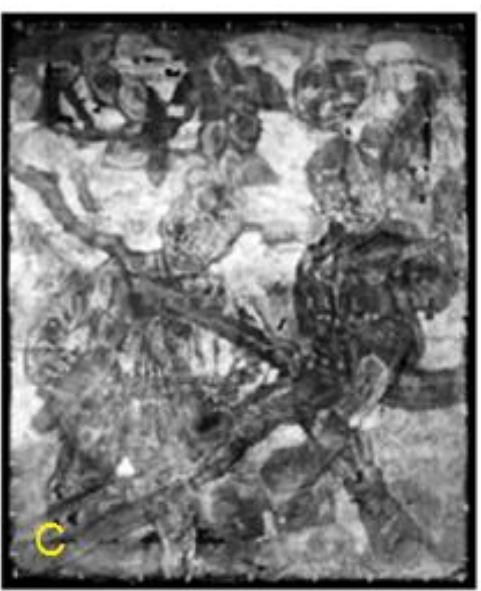

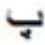

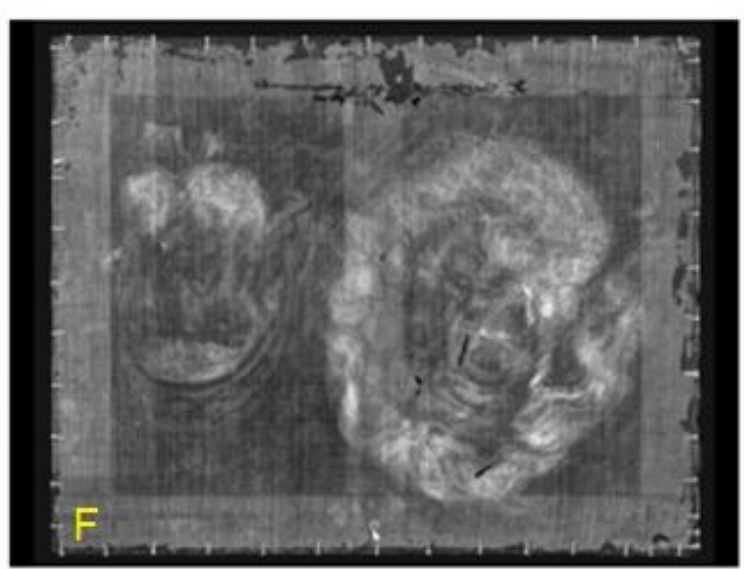

ج

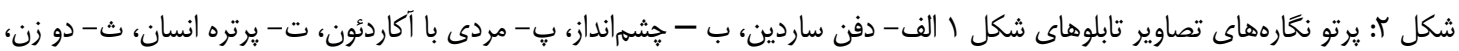

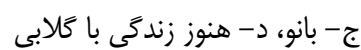

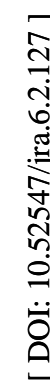

Fig. 2: The radiography of the Paintings in Fig. 1: A) The burial of the sardine, B) Scenery, C) Man with Accordion, by F) Lady G) Still Life with Pears by Valentine Durban Genaro Lahuerta, D) Portrait of man, E) Two ladies, 
جدول ( : مشخصات يرتونگارى تابلوها

Table 1. The parameters of exposure for the painting

\begin{tabular}{|c|c|c|c|c|c|}
\hline Name of painting & Detector type & $\mathrm{SFD}(\mathrm{cm})$ & time (Second) & $\begin{array}{c}\text { electric current } \\
(\mathrm{mA})\end{array}$ & voltage $(\mathrm{kV})$ \\
\hline $\begin{array}{l}\text { The burial of the } \\
\text { sardine, B-, }\end{array}$ & CR & 300 & 3 & 20 & 51 \\
\hline Scenery & Film & 120 & 1265 & 20 & 36 \\
\hline $\begin{array}{l}\text { Man with Accordion, } \\
\text { by Genaro Lahuerta, }\end{array}$ & Film & 190 & 600 & 20 & 36 \\
\hline Portrait of man, & CR & 200 & 3 & 20 & 37 \\
\hline Two ladies, & $\mathrm{CR}$ & 90 & 3 & 20 & 59 \\
\hline Lady & $\mathrm{CR}$ & 90 & 3 & 20 & 35 \\
\hline $\begin{array}{l}\text { Still Life with Pears by } \\
\text { Valentine Durban }\end{array}$ & Film & 196 & 175 & 20 & 32 \\
\hline
\end{tabular}

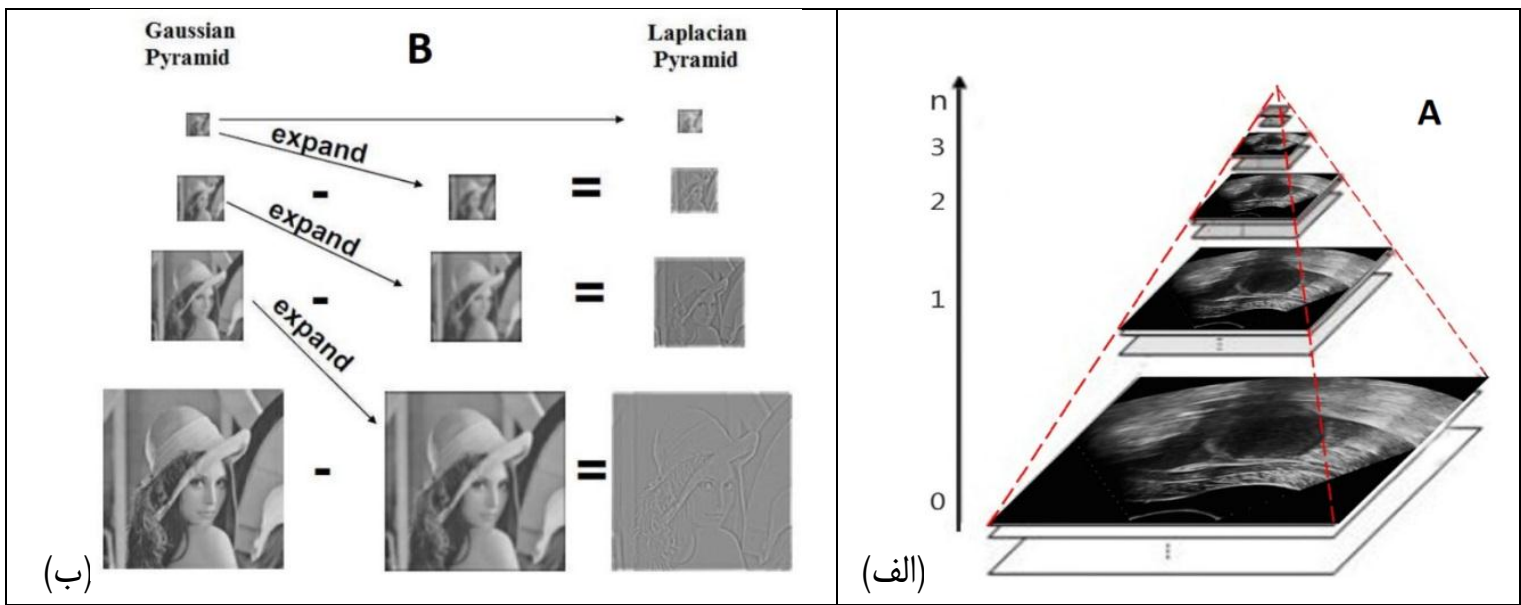

شكل س: الف: يك هرم تصوير [28]، ب: ارتباط بين هرمهاى گوسى و لإِلاسى [29]

Fig. 3: A) An image pyramid [28], B) Relationship between Gaussian and Laplacian Pyramids [29]

ســح

مى شود [29]

$L_{\ell}=g_{\ell}-\operatorname{expand}\left(g_{\ell+1}\right)$

كه در آن درايdهاى

$$
\text { تعريف مىشود: }
$$

$\operatorname{expand}\left(g_{\ell+1}(i, j)\right)=$

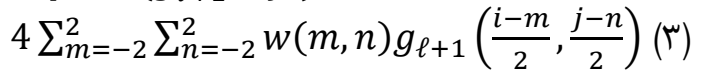

بنابراين تصوير نوفهزدايى شده

$$
\text { زير به دست آورد: }
$$

$\hat{g}_{0}=L_{0}+\operatorname{expand}\left(g_{1}\right)$.

در اين تحقيق براى افزايش كنتراسـت و شناسـايى تصاوير ينهان، از تكنيك حذف زمينهُ تصوير استفاده شد؛

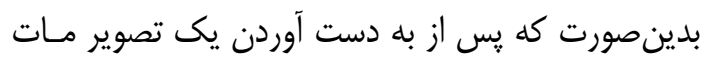

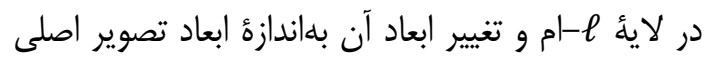
I w
فيلتر اعمال و سيس رويكرد نمونه كاهى استفاده مىشود. درنتيجه در زمان افزايش ابعاد، كه با رويكرد نمونهافزايى

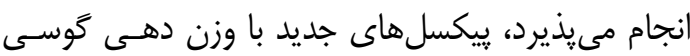
به يِيكسل هاى قبلى ساخته مى شوند.

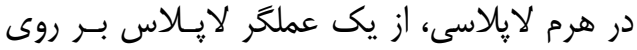

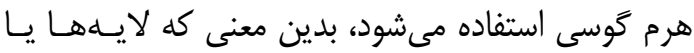

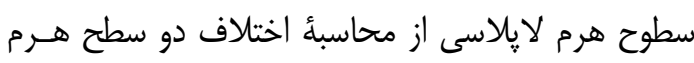

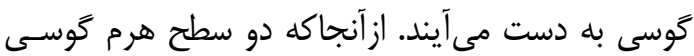

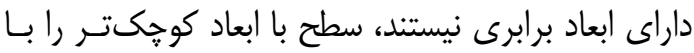

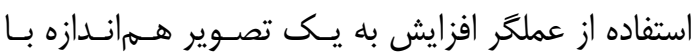

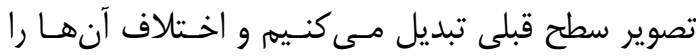
بهعنوان يك سطح از هرم لإيلاسى در نظر مسى يـيـريه، شكل س-ب را ملاحظه كنيد. بلهور دقيقتـر اخـر gl+1 
اصلى I و تصوير بعد از حذف نوفه دو داراى ابعاد M 1 د هستند، دادهشده باشند. PSNR

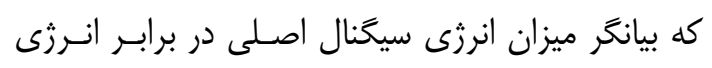

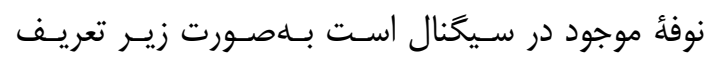

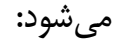
PSNR $=\frac{1}{\mathrm{MN}} \log \left(\frac{\mathrm{MN}}{\sum_{\mathrm{i}=1}^{\mathrm{M}} \Sigma_{\mathrm{j}=1}^{\mathrm{N}}\left(\mathrm{I}(\mathrm{i}, \mathrm{j})-\mathrm{I}_{\text {denoised }}(\mathrm{i}, \mathrm{j})^{2}\right.}\right)$ (ه) مقدار بيشتر PSNR نشاندهندة نزديكتـر بـودن

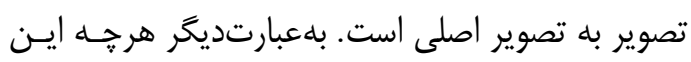

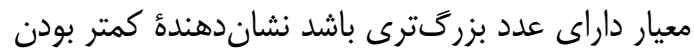

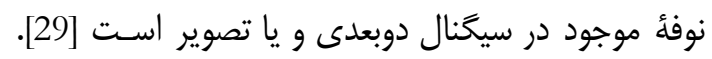

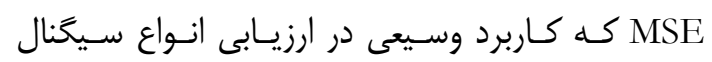

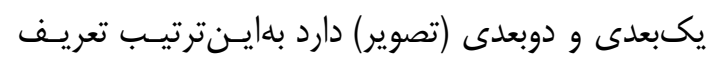
MSE $=\sqrt{\frac{1}{\mathrm{MN}} \sum_{\mathrm{i}=1}^{\mathrm{M}} \sum_{\mathrm{j} 1}^{\mathrm{N}}\left(\mathrm{I}(\mathrm{i}, \mathrm{j})-\mathrm{I}_{\text {denoised }}(\mathrm{i}, \mathrm{j})\right)^{2}}$ (7) مقدار كمتر MSE نشاندهندة شـباهت تصـوير بـهـ تصوير اصلى است.

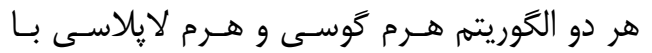

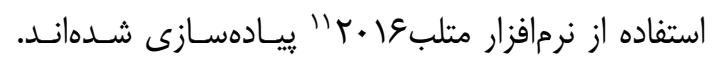

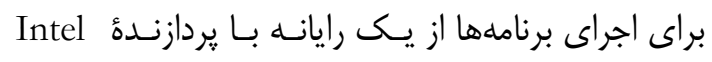
8 GB و و حافظـــ Core i7-7500U 3.6 GHz

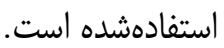

\section{ع. نتايج و يافتهها}

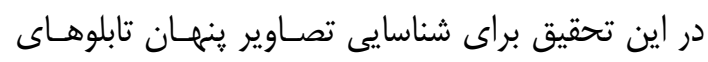

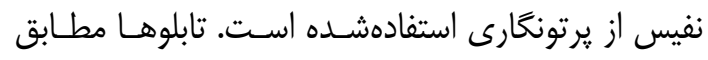

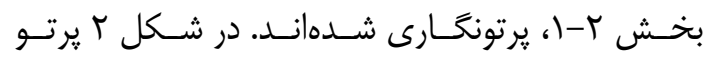

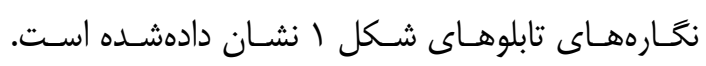

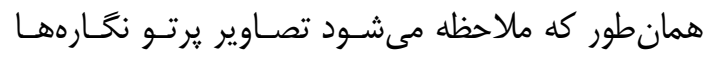

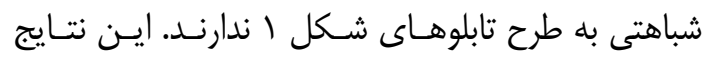
نشان مىدهند كه به علت مسائل مختلف مانند تغييرات التهات اجتماعى و سياسى جامعل، كمبـود امكانـات و مـواد، اثر ديغرى روى تابلوها كشيده شده است. در بعضى از موان موارد

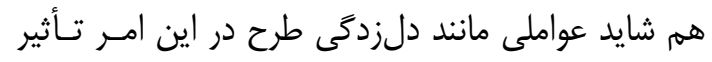

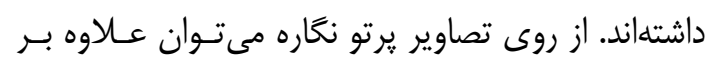

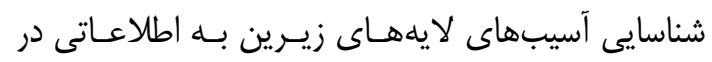

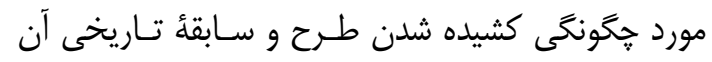

$$
\begin{aligned}
& \text { II } \\
& \text { بـا وضـوح بيشـتر }
\end{aligned}
$$

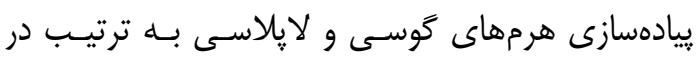

$$
\begin{aligned}
& \text { الكوريتمهاى ا و r خلاصه شده است. } \\
& \text { الخوريتم ا: الخوريتم هرم گَوسى }
\end{aligned}
$$

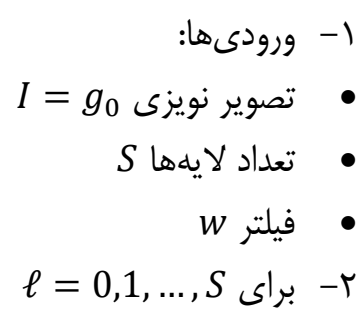

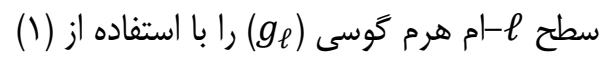

$$
\text { محاسبه كن. }
$$

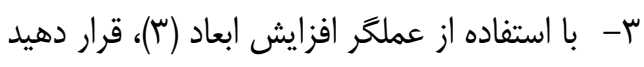

$$
\hat{g^{\wedge} 0}=\operatorname{expand}\left(g_{-} S\right)
$$$$
\text { r برار دهيد }
$$$$
I_{\text {denoised }}=I-\hat{g}_{0} \text {. }
$$

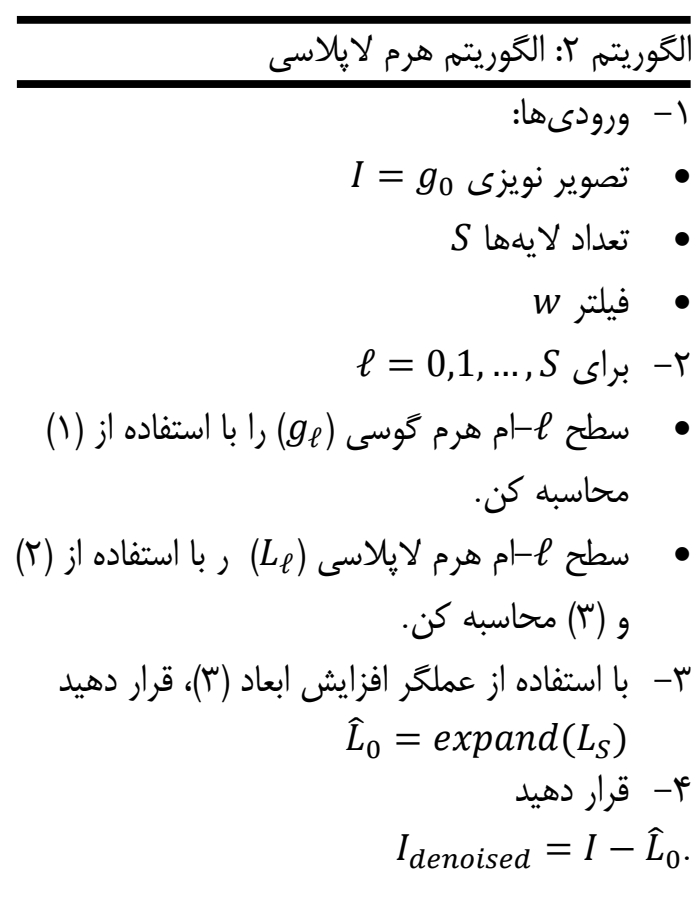

براى ارزيابى تصاوير ايجادشده، از نسـبت بيشـترين

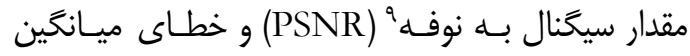
مربعى ' (MSE) استفادهشده است. فرض كنـيم تصـوير 
شكل r-الف نشان دادهشه است. براى بررسى اثر تعداد

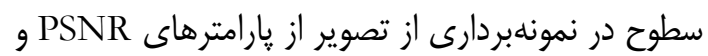

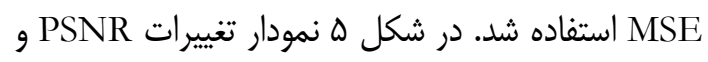

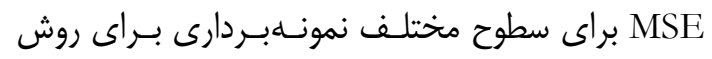

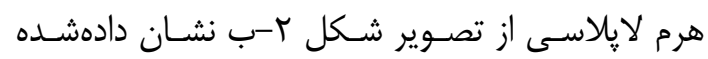

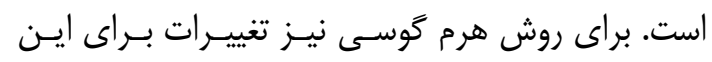

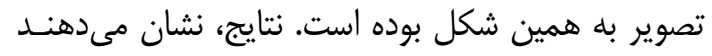
كه تغييرات PSNR و MSE هر دو صعودى هستند ولى نه از حدود ه سطح به بالا تغييرات كمتر مى شوند.

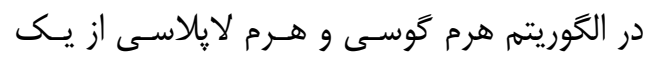

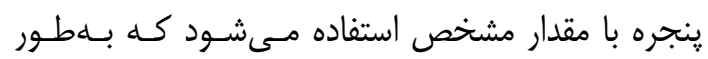

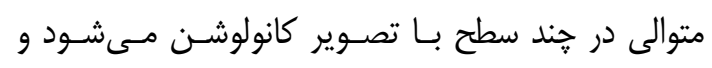

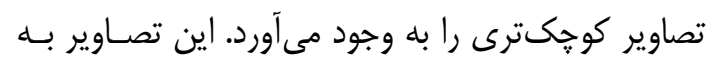

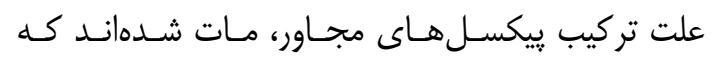

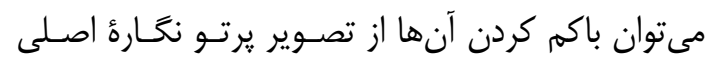

دست ييدا كرد. درروى يرتو نكارههاى شكل r علاوه بر

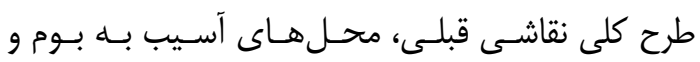

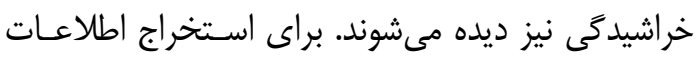

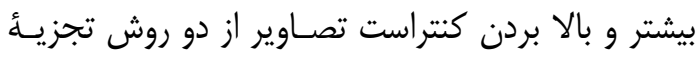

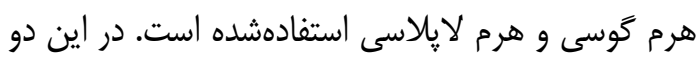

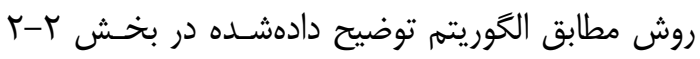

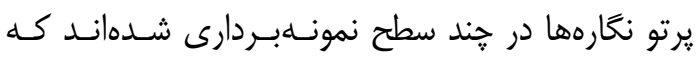

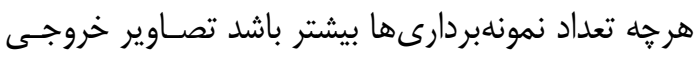

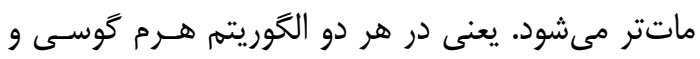

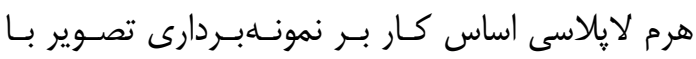

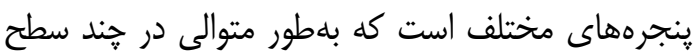
انجام و يك ينجره در هر مرحله در تصوير درهمبييجيــاه

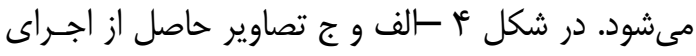

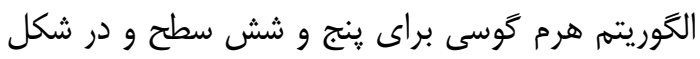

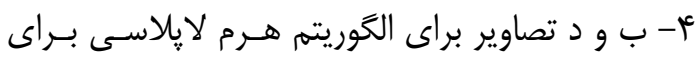

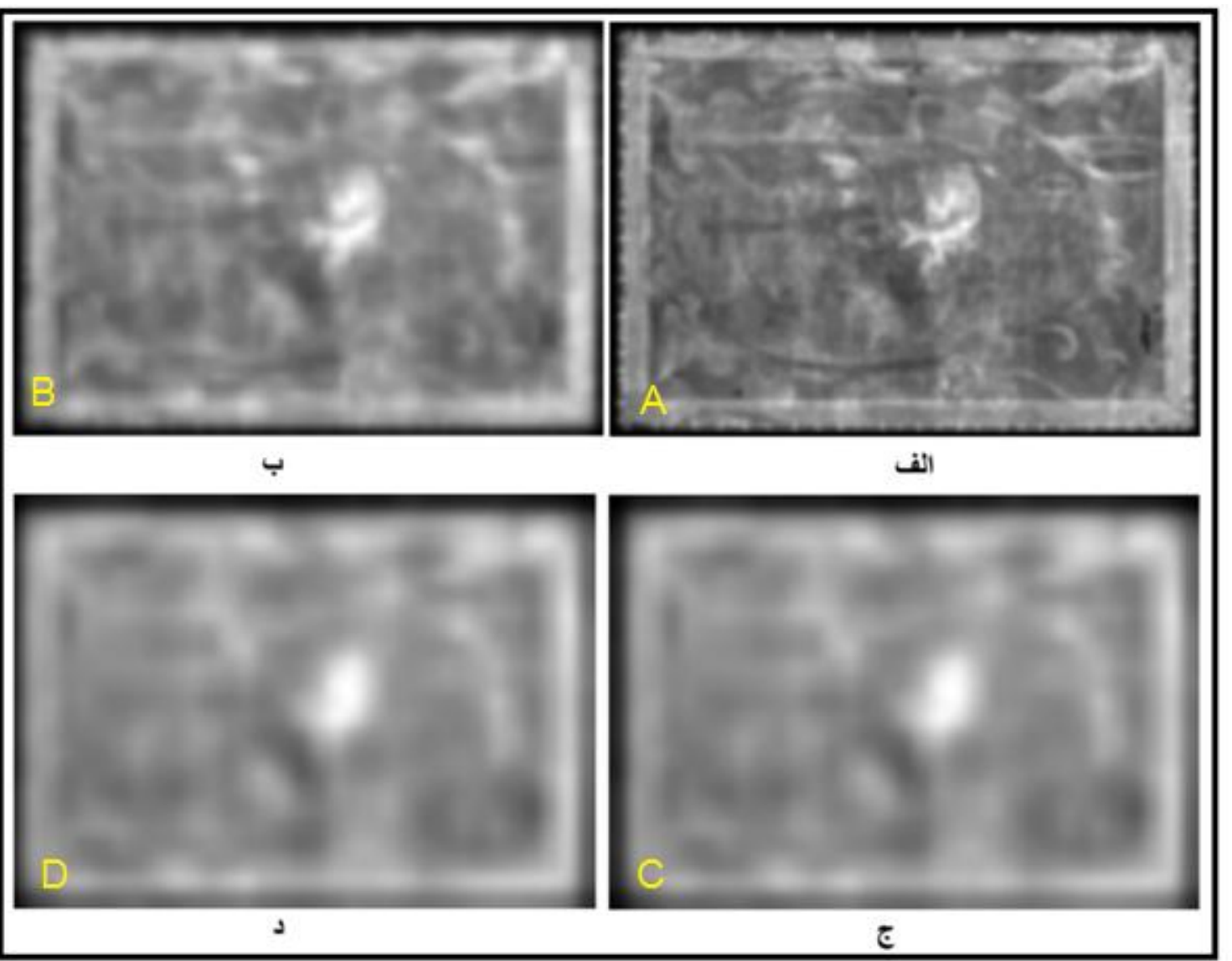

شكل ع: تصوير خروجى (براى شكل r- الف): الف و ج- روش هرم گوسى ب و د- روش هرم لإِلاسى براى نمونهبردارى در سطوح ه و Fig. 4: Output image (for Fig.2-A): A and C) Gaussian pyramid method, B and D) Laplace pyramid method for sampling at levels 5 and 6 

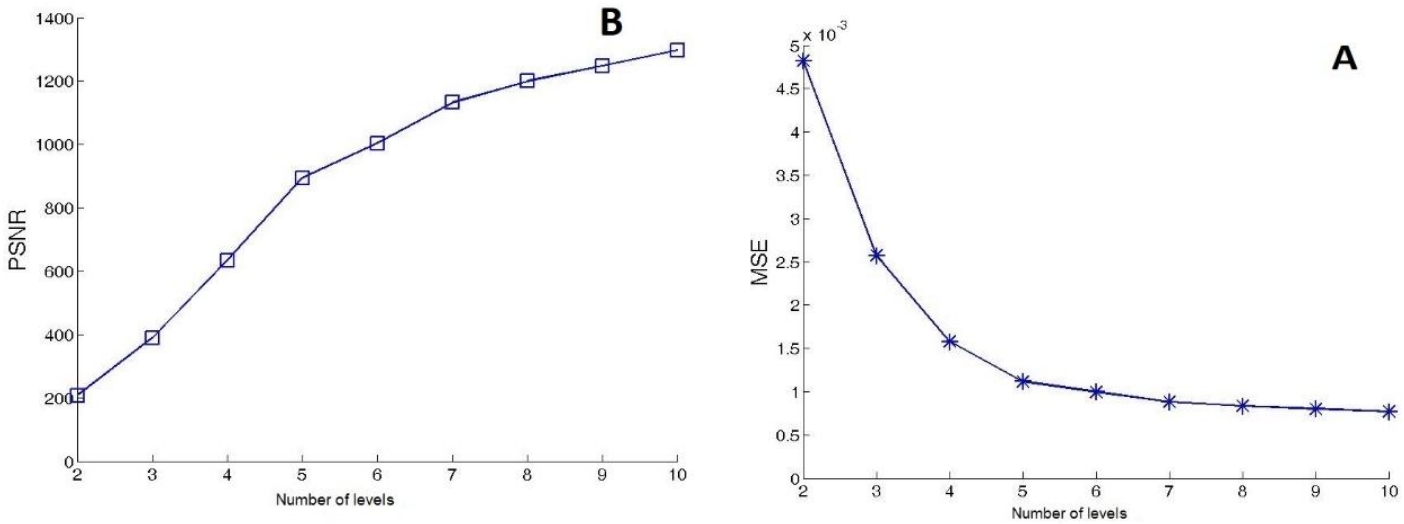

ب

الف

شكل ه. نمودار تغييرات الف- MSE و ب- PSNR برحسب سطح براى شكل r- الف براى روش هرم لإلاسى ب

Fig. 5. The variation of A) MSE and B) PSNR with different level for Fig. 2-A for the Laplacian pyramid method.

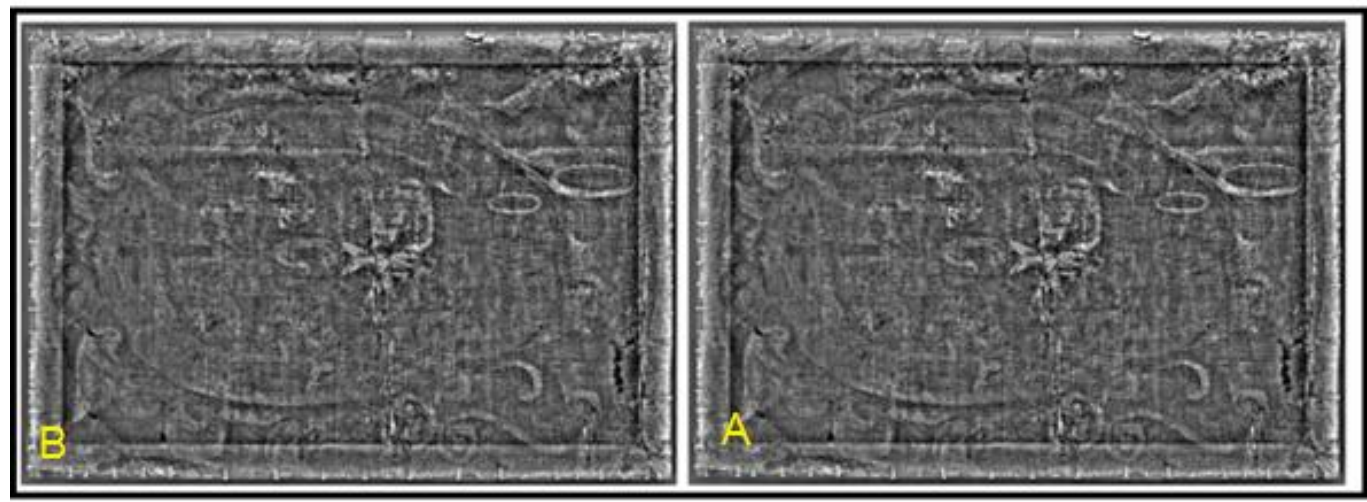

ب

الف

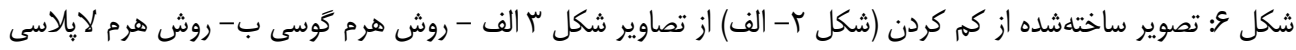

Fig. 6: The reconstructed image by subtracting (Fig. 2- A) from the images Fig. 3 A) Gaussian pyramid method, B) Laplacian pyramid method.

نشان دادهشده است. مسير كشيده شدن قلـممـو، نـواحى

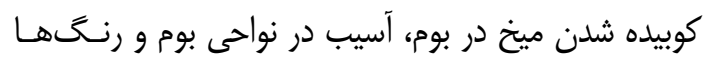

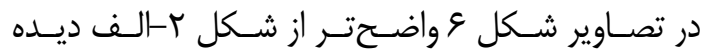

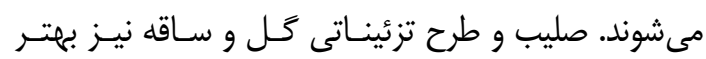

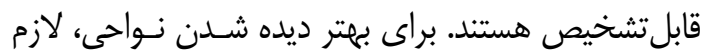

$$
\text { است آنها را با بزرخنمايى بالا بيينيد. }
$$

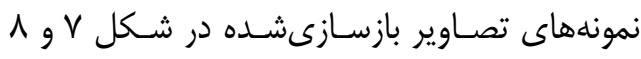

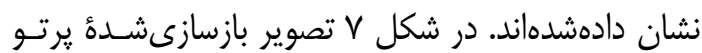

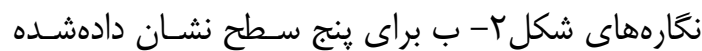

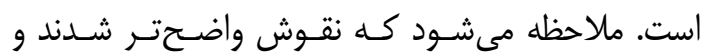

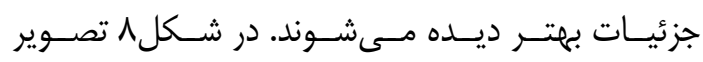

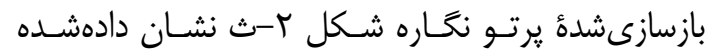

است.
تصــاويرى بــا وضـوح بهتــر بـــه دسـت آورد. بررسـى

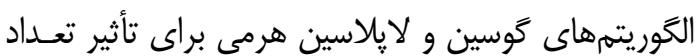

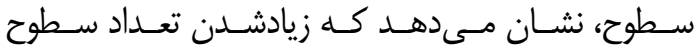

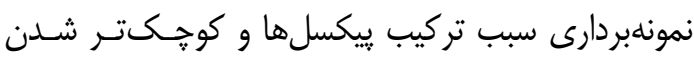
تصاوير مىشود كه اخر تصاوير، در اندازه تصوير اوليه ديده

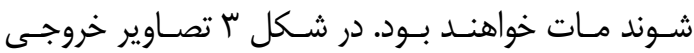

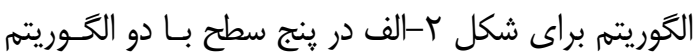
هرم گوسى و هرم لايلاسى نشان دادهشده است. مشاهده

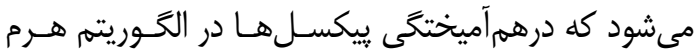
لإِلاسى بيشتر است و تصوير ماتتر شده است. در شكل دهل

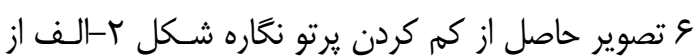

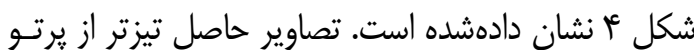

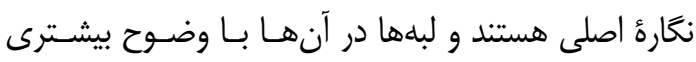




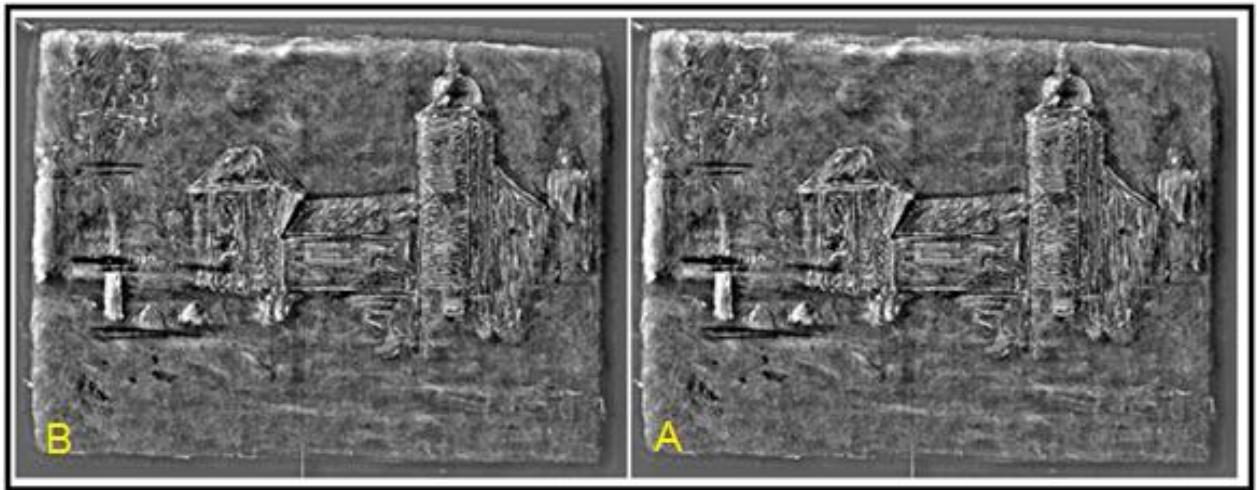

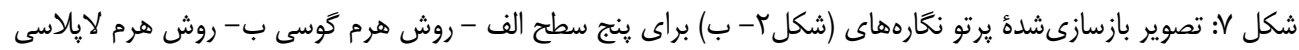
Fig. 7: The reconstructed image of radiographs (Fig. 2-b) for five levels, A) The Gaussian pyramid method, B) the Laplacian pyramid method

تابلوهاى مردى با آكاردئون و يرترء انسان بعد از يردازش

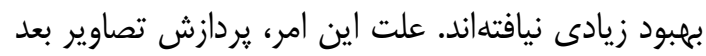

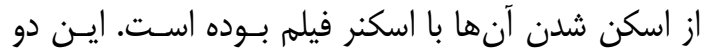

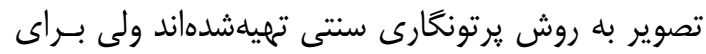

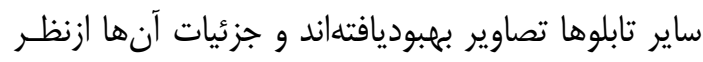

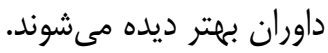

براى ارزيابى نتايج حاصل، تصاوير بازسازىشده به رؤيت

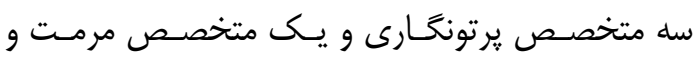

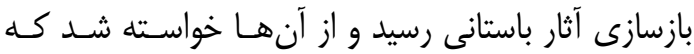

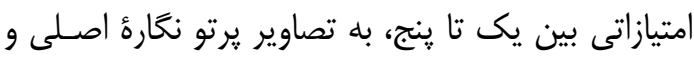
تصاوير بازسازىشده با دو روش، اختصاص دهند. نتـايج

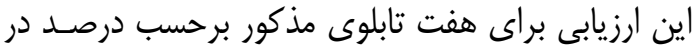
جدول r ثبتشده است. بررسى نتايج نشان مى بدهد كـهـ

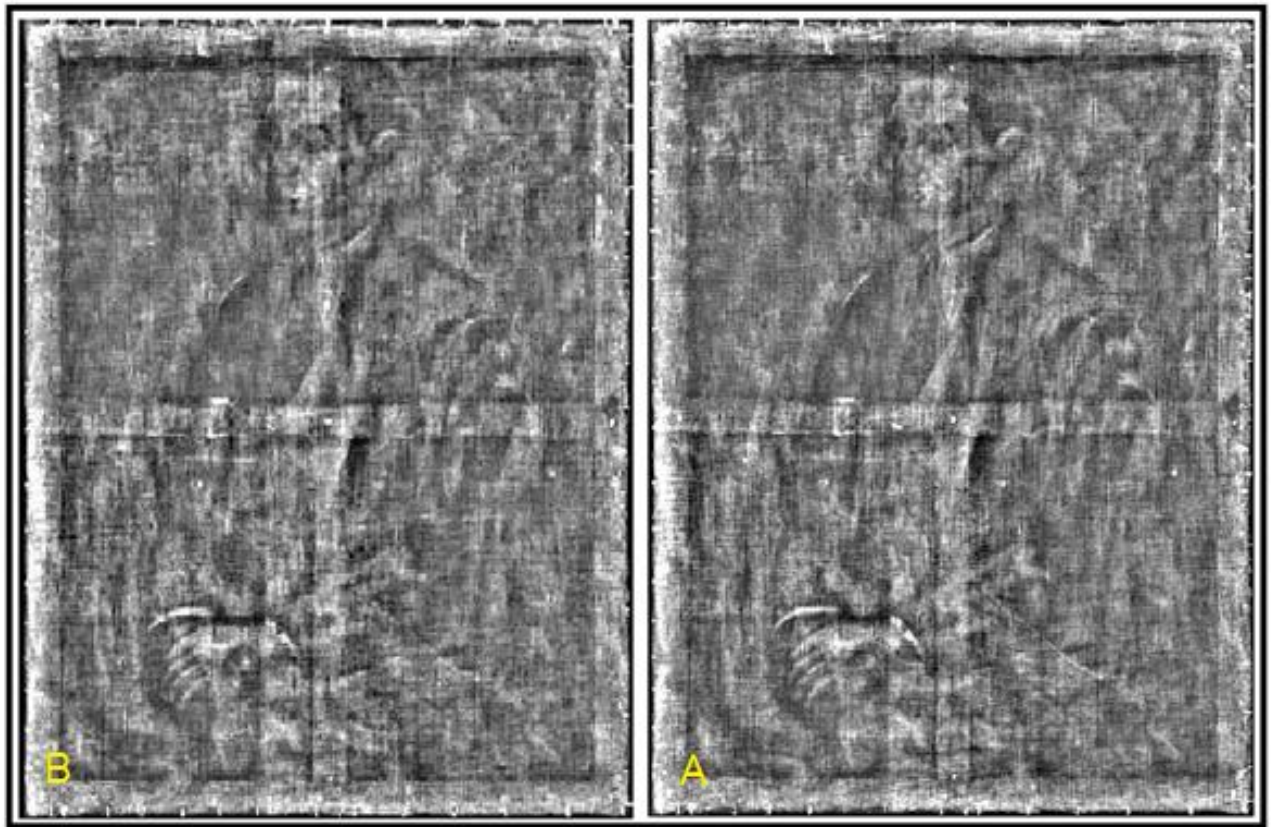

ب

الف

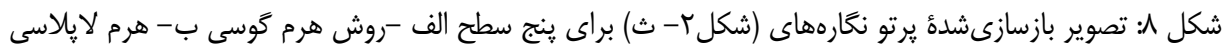

Fig. 8: The reconstructed image of radiographs (Fig. 2-c) for the five levels A) Gaussian pyramid method, B) Laplace pyramid

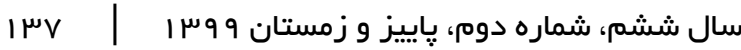


جدول r: درصد امتيازات متخصصان به يرتو نگًارههاى اصلى و بازسازىشده با دو روش هرم گوسى و هرم لايلاسى

Table 2: Percentage of experts' scores on original and reconstructed radiographs by Gaussian pyramid and Laplace pyramid methods

\begin{tabular}{|c|c|c|c|}
\hline Name of painting & $\begin{array}{c}\text { Original } \\
\text { radiograph }\end{array}$ & $\begin{array}{c}\text { Reconstructed by } \\
\text { Gaussian Pyramid } \\
\text { method }\end{array}$ & $\begin{array}{c}\text { Reconstructed by } \\
\text { Laplacian Pyramid } \\
\text { method }\end{array}$ \\
\hline The burial of the sardine, B-, & $68 \%$ & $92 \%$ & $91 \%$ \\
\hline Scenery & $72 \%$ & $93 \%$ & $94 \%$ \\
\hline Man with Accordion, by Genaro Lahuerta, & $92 \%$ & $92 \%$ & $92 \%$ \\
\hline Portrait of man, & $93 \%$ & $92 \%$ & $91 \%$ \\
\hline Two ladies, & $81 \%$ & $96 \%$ & $96 \%$ \\
\hline Lady & $81 \%$ & $96 \%$ & $95 \%$ \\
\hline Still Life with Pears by Valentine Durban & $79 \%$ & $91 \%$ & $91 \%$ \\
\hline
\end{tabular}

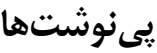

1. Multi Spectral Imaging

2. Scanning Electron Microscope Equipped with X-ray energy Diffraction Spectroscopic 3. analysis

4. Non-destructive testing or Nondestructive evaluation

5. Computed Radiography

6. Phosphor plate

7. Noise

8. Down-sampling

9. Up-sampling

10. Peak Signal to Noise Ratio

11. Mean Square Error

12. MATLAB

\section{ه. نتيجهل}

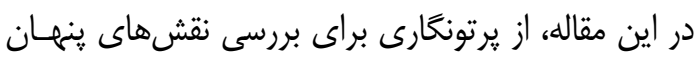
تابلوهاى اثرى استفادهشده است. بررسى ها نشان مي دهند برسي

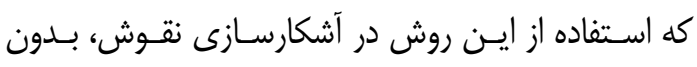

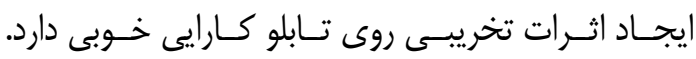

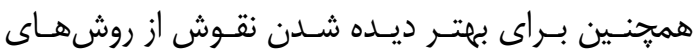

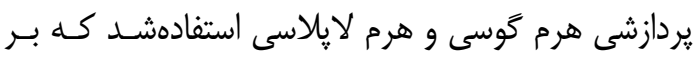

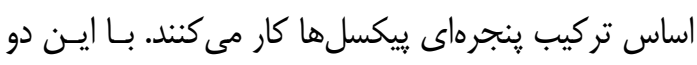

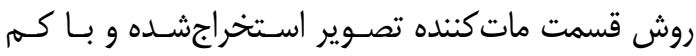

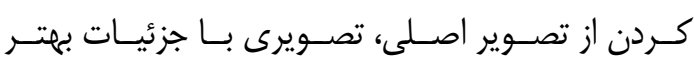

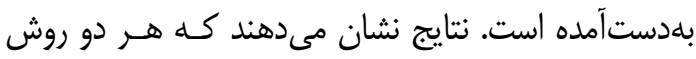

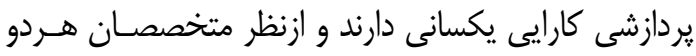
به يك اندازه در بهبود تصوير تأثير دارند.

method in art analysis. Canadian Journal of Physics. 2014; 92(4):341-64.

[4] Ghanooni M. Report on the maintenance and restoration of the painting of the camp by the river by Kamal Ol-Molk. Library, Museum and Document Center Islamic Consultative Assembly, 2019.

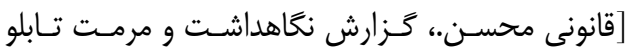

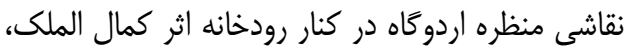

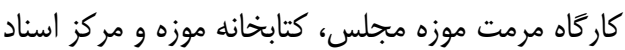

مجلس شوراى اسلامى؛ موسז ا.]

[5] Afzali N, Vatan-Parast R. Pathology and erosion analysis of oil paintings on Kamal-ol-Molk canvas in Golestan Palace. Athar Journal. Spring 2017, 38(76): 3-76.

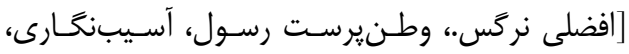


آسيبشناسى و تحليـل فرسـودگى هــاى نقاشـىهـاى رنغ روغن روى بوم كمال الملك در كاخ كلستان، اثر

فصلنامه علمى فنى هنرى؛ بهار عوس رسا.]

[6] Baronti S, Casini A, Lotti F, Porcinai S. Multispectral imaging system for the mapping of pigments in works of art by use of principal-component analysis. Applied optics. 1998 Mar 10; 37(8):1299309.

[7] Casini A, Lotti F, Picollo M, Stefani L, Buzzegoli E. Image spectroscopy mapping technique for noninvasive analysis of paintings. Studies in conservation. 1999 Jan 1; 44(1):39-48.

[8] Balas C, Papadakis V, Papadakis N, Papadakis A, Vazgiouraki E, Themelis G. A novel hyper-spectral imaging apparatus for the non-destructive analysis of objects of artistic and historic value. Journal of Cultural Heritage. 2003 Jan 1; 4: 330-7.

[9] Fischer C, Kakoulli I. Multispectral and hyperspectral imaging technologies in conservation: current research and potential applications. Studies in Conservation. 2006 Jun 1; 51(sup1):3-16.

[10] Vilaseca M, Pujol J, Arjona M, de Lasarte M. Multispectral system for reflectance reconstruction in the near-infrared region. Applied optics. 2006 Jun 20; 45(18):4241-53.

[11] Cristoforetti G, Legnaioli S, Palleschi V, Salvetti A, Tognoni E. Optical chemical sensors for cultural heritage. InOptical Chemical Sensors 2006 (pp. 515-526). Springer, Dordrecht.

[12] Bonifazzi C, Carcagnì P, Fontana R, Greco M, Mastroianni M, Materazzi M, Pampaloni E, Pezzati L, Bencini D. A scanning device for VIS-NIR multispectral imaging of paintings. Journal of Optics A: Pure and Applied Optics. 2008 May 1; 10 (6):064011.

[13] Arbabi F. Restoration of several pins excavated from Salmabad village in Khosf (South Khorasan). Bi-annual Journal of Restoration Science and Culture Heritage; Spring 2018, 5(9):1-11. [in Persian]

[اربابى فائزه، مرمت جند نمونه سنجاق مكشوفه از سلهم

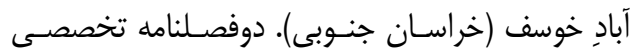

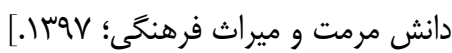

[14] Taft WS, Mayer JW. The science of paintings. Measurement Science and Technology, 2001.

[15] Berezhnoy IE, Postma EO, van den Herik HJ. Authentic: computerized brushstroke analysis. In2005 IEEE International Conference on Multimedia and Expo 2005 Jul 6 (pp. 1586-1588).

[16] B. Raj, "Practical N.D.T.", Alpha Science, 3th edition, 2007.

[17] Cortz L. Non-Destructive Testing. ASM International; 1995.

[18] Toyserkani H. Nondestructive evaluations, Jahad Daneshgahi, Isfahan, 2015. [in Persian]

[تويسر كانى ح.، بررسىهاى غيرمخرب. اصفهان: جهاد

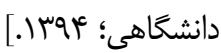

[19] Bridgman CF. The amazing patent on the radiography of paintings. Studies in Conservation. 1964 Nov 1; 9(4):135-9.

[20] Nacereddine N, Drai R, Benchaala A. Weld defect extraction and identification in radiograms based neural networks. InProc. IASTED International Conference on Signal Processing, Pattern Recognition, and Applications, Crete, Greece 2002 Jun 25 (pp. 38-43).

[21] Movafeghi A, Kargarnovin MH, Soltanian-Zadeh H, Taheri M, Ghasemi F, Rokrok B, Edalati K, Rastkhah N. Flaw detection improvement of digitised radiographs by morphological transformations. Insight-NonDestructive Testing and Condition Monitoring. 2005 Oct 1; 47(10):625-30.

[22] Felisberto MK, Lopes HS, Centeno TM, De Arruda LV. An object detection and recognition system for weld bead extraction from digital radiographs. Computer Vision and Image Understanding. 2006 Jun 1; 102(3):23849.

[23] Daubechies I, Defrise M, De Mol C. An iterative thresholding algorithm for linear inverse problems with a sparsity constraint. Communications on Pure and Applied Mathematics: A Journal Issued by the Courant Institute of Mathematical Sciences. 2004 Nov; 57(11):1413-57.

[24] Carrasco MA, Mery D. Segmentation of welding defects using a robust algorithm. Materials Evaluation. 2004; 62(11):11427.

[25] Carvalho AA, Suita RC, Silva RR, Rebello 
JM. Evaluation of the relevant features of welding defects in radiographic inspection. Materials Research. 2003 Jun; 6(3):427-32.

[26] Lampert CH, Blaschko MB, Hofmann T. Efficient subwindow search: A branch and bound framework for object localization. IEEE transactions on pattern analysis and machine intelligence. 2009 Jul 17; 31(12):2129-42.

[27] Mery D, Riffo V, Zuccar I, Pieringer C. Automated X-ray object recognition using an efficient search algorithm in multiple views. In Proceedings of the
IEEE conference on computer vision and pattern recognition workshops 2013 (pp. 368-374).

[28] Liao X, Yuan Z, Zheng Q, Yin Q, Zhang D, Zhao J. Multi-scale and shape constrained localized region-based active contour segmentation of uterine fibroid ultrasound images in HIFU therapy. PloS one. 2014 Jul 25; 9(7):e103334.

[29] Leavline EJ, Sutha S. Design of FIR Filters for Fast Multiscale Directional Filter Banks. International Journal of uand e-Service, Science and Technology. 2014; 7(5):221-34. 